\title{
Elektrokapillarkurven in nichtwässerigen Lösungen
}

\section{Doctoral Thesis}

Author(s):

Wild, J.Heinrich

Publication date:

1923

Permanent link:

https://doi.org/10.3929/ethz-a-000091973

Rights / license:

In Copyright - Non-Commercial Use Permitted 


\title{
Elektrokapillarkurven in nichtwässerigen Lösungen
}

\author{
a.re
cieg \\ VON DER \\ EIDGENÖSSISCHEN TECHNISCHEN \\ HOCHSCHULE IN ZÜRICH \\ ZUR ERLANGUNG \\ DER WÜRDE EINES DOKTORS \\ DER NATURWISSENSCHAFTEN \\ OENEHMIGTE \\ PROMOTIONSARBEIT \\ VORGELEGT VON
}

J. HEINRICH WILD

DIPL. FACHLEHRER DER NATURWISSENSCHAFTEN

AUS MITLÖDI (GLARUS)

Referent: Herr Prof. Dr. E. Baur.

Korreferent: Herr Prof. Dr. W. D. Treadwell.

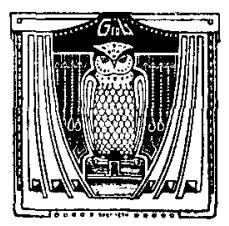

Leipzig

Druck von Breitkopf \& Haertel

1923 


\title{
Elektrokapillarkurven in nichtwässerigen Lösungen.
}

\author{
Von \\ H. Wild. \\ (Mit 19 Figuren im Text.) \\ (Eingegangen am 30. 7. 22.)
}

Die Aufgabe.

In der Arbeit von E. Baur und S. Kronmann „Über die Ionenadsorptionspotentiale" 1) wird darauf hingewiesen, dass man über die Grösse des Potentialsprunges an der Grenze zweier unvollständig mischbarer Flüssigkeiten, wenn Verteilungsgleichgewicht besteht, auf eine bestimmte Weise Aufschluss erhalten kann. Betrachtet man nämlich die Kette

$$
\begin{aligned}
& M|M S| M S \mid M \\
& \text { Phase I Phase II }
\end{aligned}
$$

$$
\pi_{1} \quad \pi_{3} \quad \pi_{2}
$$

wo $M$ ein Metall bedeutet und $M S$ ein Salz dieses Metalls, das in der Phase I und in der Phase II in solchen Konzentrationen aufgelöst ist, dass die beiden Phasen in bezug auf das Salz im Verteilungsgleichgewicht stehen, so ist, vorausgesetzt, dass auch die beiden Lösungsmittel selbst sich untereinander in das Verteilungsgleichgewicht gesetzt haben, die elektromotorische Kraft der Kette Null, da ja eine kleine elektrolytische Verschiebung an dem chemischen Zustand nichts ändern, in dem Gebilde also auch keine elektrische Energie aus chemischer entstehen kann ${ }^{2}$ ) Es ist sonach

$$
\pi_{1}-\pi_{2}+\pi_{3}=0
$$

1) E. Baur und S. Kronmann, Zeitschr. f. physik. Chemie 92, 81 (1916).

2) Vgl. R. Luther, Zeitschr. f. physik. Chemie 19, 567 (1896). 
Sind wir nun imstande, $\pi_{1}$ und $\pi_{2}$ einzeln $\mathrm{zu}$ bestimmen, so könnte die Phasengrenzkraft $\pi_{3}$ sogleich ausgewertet werden. Mittel, um Einzelpotentialsprünge zu messen, stehen uns in der Verwertung von Elektrokapillarkurven zur Verfügung. Dieses Hilfsmittel ist allerdings zwei Beschränkungen unterworfen.

Man ist erstens in der Praxis auf Quecksilber als metallische Phase angewiesen, da bei Amalgamen Verwicklungen eintreten ${ }^{1}$, die der Deutung der bezüglichen Elektrokapillarkurven Schwierigkeiten entgegenstellen. Diese Einschränkung ist indessen für unseren Zẃeck nicht besonders hinderlich, da man ja den Phasen I und II ausser dem passenderweise schwerlöslich zu wählenden Quecksilbersalz noch beliebige andere Salze zusetzen kann, so lange sie nur mit Quecksilber chemisch verträglich sind.

Wesentlicher ist die zweite Beschränkung, der wir in der Auswahl der zu lösenden Salze unterworfen sind. Aus den ausgedehnten Untersuchungen Gouy's ${ }^{2}$ ) und aus neuerlichen ergänzenden Messungen von $H$. Freundlich und M. Wreschner ${ }^{3}$ weiss man, dass die Lippmannsche Elektrokapillarkurve Deformationen erfährt, wenn in der wässerigen Lösung, die an die kapillare Quecksilberkuppe angrenzt, sogenannte "kapillaraktive" Ionen vorhanden sind. Es sind dies solche Ionen, die, wie wir aus anderweitiger Erfahrung wissen, an Phasengrenzen in ausgeprägter Weise adsorbiert werden ${ }^{4}$ ). Indem sich über die Nernstsche Doppelschicht die Doppelschicht der Ionenadsorption lagert, wird die von der ersteren bestimmte Lage des Maximums der Lippmann-Kurve nach der Seite einer tieferen oder höheren Polarisation verschoben, je nachdem es sich um die Adsorption eines Kations oder Anions handelt.

Es ist einleuchtend, dass in solchen Fällen das Maximum nicht mehr zusammenfällt mit dem isoelektrischen Punkt und dann ungeeignet ist, um aus ihm den Betrag des Nernst schen Potentialsprunges $\pi_{1}$ oder $\pi_{2} \mathrm{zu}$ bestimmen. Ich hatte mich daher bei der Auswahl der zwischen den beiden Lösungsphasen zu verteilenden Salzen auf solche zu beschränken, die nach bisheriger Erfahrung möglichst wenig kapillaraktiv sind.

Die Frage, ob die dann zu erhaltenden Maxima der LippmannKurve den isoelektrischen Punkt getreu wiedergeben, darf man heute

\footnotetext{
1) V. Rothmund, Zeitschr. f. physik. Chemie 15, 1 (1894).

2). Go u y, Ann. de Chim. et de Phys. (7) 29, 145 (1903); (8) 8, 291 (1906); (8) 9, 75 (1906).

3) H. Freundlich und M. Wreschner, Kolloidzeitschr. 28, 250 (1921).

4) Vgl. H. Freundlich, Kapillarchemie, 2. Aufl., S. 397-406, Leipzig 1922.
} 
wohl unbedenklich bejahen. Die beiden neuesten lehrbuchmässigen Darstellungen der Elektrokapillarität, die von M. Trautz im Graetzschen Handbuch der Elektrizität und des Magnetismus $\left.{ }^{1}\right)$ und die von H. Freundlich in seiner Kapillarchemie ${ }^{2}$, kommen übereinstimmend zum Schlusse, dass in einigen, jetzt gut bekannten Fällen, vorweg in Lösungen von Kaliumchlorid, Kaliumnitrat und Schwefelsäure, das Maximum der Lippmann-Kurve erlaubt, den absoluten Betrag eines Nernstschen Einzelpotentialsprunges zutreffend zu bestimmen. Nachdem Nernst ${ }^{3}$ ) gezeigt hatte, dass die Grenzflächenspannung des Quecksilbers in der Tat mit der Mercuroionkonzentration in der von der Theorie geforderten Weise sich ändert, waren es wohl hauptsächlich die gute von Krumreich 4 ) erzielte Übereinstimmung mit der Tropfelektrode und der nach F. Krüger und Krumreich5) fast völlig exakte Anschluss der gegen Kaliumnitratlösung aufgenommenen Elektrokapillarkurve an die Formel von G. Lippmann und Helmholtz, welche das Zutrauen in die Gleichsetzung von isoelektrischem Punkt und Maximum der Lippmann-Kurve festigten. Hiervon darf also als von einer fest begründeten und nicht mehr umstrittenen Tatsache ausgegangen werden.

Man durfte es daher unternehmen, den vorerwähnten Flüssigkeitspotentialsprung $\pi_{3} \mathrm{zu}$ bestimmen, indem Elektrokapillarkurven aufgenommen wurden gegen eine möglichst grosse Zahl von paarweise im Verteilungsgleichgewicht stehenden Flüssigkeiten. Die Flüssigkeiten mussten solche Salze enthalten, mit denen man in Wasser die richtigen Kurven und Maxima bekommt; also kommen zunächst Chlorkalium und Kaliumnitrat in Betracht, aber auch Mercuronitratlösung mit Salpetersäure angesäuert, womit F. Krüger und Krumreich ${ }^{6}$ ) ebenfalls brauchbare Messungen erhalten hatten.

Über Elektrokapillarität gegen nichtwässerige Lösungen ist nicht viel bekannt. Immerhin ergibt eine Durchsicht der Literatur, dass es nicht aussichtslos erscheint, auch gegen nichtwässerige Lösungen ordentliche Kurven $\mathrm{zu}$ bekommen. Nachdem zuerst Campetti ${ }^{7}$ ) sich

1) Bd. I, Abschnitt Galvanische Elemente, S. 695, 698, Barth, Leipzig 1918.

2) H. Freundlich, 2. Aufl., S. 396, Akadem. Verlagsges., Leipzig 1922.

3) Nernst, Zeitschr. f. Elektrochemie 4, 29 (1897).

4) Krumreich, Zeitschr. f. Elektrochemie 19, 622 (1913).

5) F. Krüger und Krumreich, Zeitschr. f. Elektrochemie 19, 622 (1913).

6) F. Krüger und Krumreich, a. a. O.

7) Campetti, Atti della Acead. delle Sc. di Torino 29, 62, 228 (1893), zitiert nach

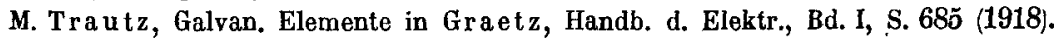


mit alkoholischen Lösungen befasst hatte, verwendete Krumreich ${ }^{1}$ ) sogar mit besonderem Vorteil Lösungen mit 90\% igem Alkohol. Ihnen schliessen sich Messungen von E. Newbery ${ }^{2}$ ) an mit an Salz gesättigten, absolut alkoholischen Lösungen, die zum Ausgangspunkt für meine Arbeit wurden. Zuvor hatte schon Gouy in seiner dritten Arbeit aus dem Jahre $1906^{3}$ ) orientierende Messungen an einer Reihe nichtwässeriger Flüssigkeiten („corps purs, additionés d'un peu d'eau“) und an Gemischen von Pyridin - Aceton, Methylalkohol - Anilin, Methylalkohol - Chloroform angestellt und gefunden, dass sich regelmässige Lippmann-Kurven an ihnen gewinnen lassen. Somit erscheint es fast auffallend, dass von diesem Hilfsmittel zur Bestimmung der Phasengrenzkräfte $\pi_{3}$ nicht schon längst ein systematischer Gebrauch gemacht worden ist. Vermutlich liess man sich von theoretischen Bedenken bezüglich der Auswertung der Kurven abhalten.

Seitdem Nernst ${ }^{4}$ ) spezifische Ionenteilungskoeffizienten $k^{+}$und $k^{-}$ (für Kation und Anion eines Salzes) eingeführt hatte, die von dem Teilungsverhältnis $k$ des undissoziierten Salzes zu unterscheiden seien, und für den daher rührenden Potentialsprung $\pi_{3}$ beim Verteilungsgleichgewicht die Formel gegeben hatte ${ }^{5}$ ):

$$
\pi_{3}=\frac{R T}{F} \ln k^{+} \frac{C_{\mathrm{I}}}{C_{\mathrm{II}}}=\frac{R T}{F^{-}} \ln \frac{1}{k^{-}} \frac{C_{\mathrm{II}}}{C_{\mathrm{I}}^{-}},
$$

wo $C_{\mathrm{I}}$ und $C_{\mathrm{II}}$ die Ionenkonzentration in der Phase I und in der Phase II bedeutet, bestand ein bestimmtes Interesse an einer Methode zur Messung von $\pi_{3}$. Allein noch 1902 mussten E. H. Riesenfeld ${ }^{6}$ ) und übereinstimmend $1906 \mathrm{E}$. Abel ${ }^{7}$ ) feststellen, dass $\pi_{3}$ der direkten Messung „zur Zeit unzugänglich“ sei. Bei Messungen von Ketten, wie die von E. Baur und Kronmann ${ }^{8}$ ) untersuchten, bekommt man entweder die Differenz zweier $\pi_{3}$ oder man muss rechts und links von der einen Phasengrenze zwei Salze verteilen, wodurch andere Verwicklungen entstehen. Demgegenüber scheint die Bestimmung von $\pi_{3}$ nach der oben angeschriebenen Gleichung aus der Differenz $\pi_{1}-\pi_{2}$ weit eindeutiger.

1) Krumreich, a. a. 0.

2) E. Newbery, Journ. Chem. Soc. London 107, 852, 1520 (1915).

3) Gouy, Ann. de Chim. et de Phys. (8) 9, 75 (1906), Kap. II und III, S. 132-134.

4) Nernst, Zeitschr. f. physik. Chemie 8, 138 (1891); 9, 137 (1892).

5) Vgl. auch E. Abel, Zeitschr. f. physik. Chemie 56, 612 (1906).

6) E. H. Riesenfeld, Ann. d. Phys. (4) 8, 616 (1902).

7) E. Abel, a. a. 0 .

8) E. Baur und Kronmann, a. a. 0. 
Man könnte noch daran denken, das $\pi_{3}$ aus der Verschiebung eines kapillaren Meniskus an der Grenze der beiden flüssigen Phasen unmittelbar zu bestimmen, wenn bei Polarisation von $\pi_{\mathbf{3}}$ eine Elektrokapillarkurve erhalten werden kann. Vorläufige Versuche, welche zeigen, dass man bei der Elektrolyse zweiphasiger Flüssigkeitsketten Verschiebungen eines kapillaren Meniskus tatsächlich erhält, rühren von Krouchkoll1) her. Doch lässt sich denselben nicht entnehmen, ob man eine Lippmann-Kurve mit ausgeprägtem Maximum verwirklichen kann. Mehrfache Verwicklungen sind zu erwarten 2), so dass es vorläufig eher geboten schien, der Messung von $\pi_{1}$ und $\pi_{2}$ sich zuzuwenden.

Es gibt eine gewisse Andeutung dafür, dass die $\pi_{3}$ Null sind, oder von Null nur wenig abweichen, oder anders ausgedrückt: dass die spezifischen Ionenlöslichkeiten oder Ionenteilungskoeffizienten nur wenig spezifisch sind, also für alle Ionen gleich oder nur wenig verschieden sind, und also im wesentlichen nur von den betrachteten Lösungsmitteln abhängen. Diese Andeutung kann aus zwei eigentümlichen empirischen Regeln geschöpft werden, die von P. Walden ${ }^{3}$ ) aufgefunden worden sind. Walden findet, dass die Ionenkonzentrationen eines gegebenen Elektrolyten - es war Tetraäthylammoniumjodid in den gesättigten Lösungen sehr verschiedener Lösungsmittel sich verhalten wie die dritten Potenzen von ihren Dielektrizitätskonstanten. Gesättigte Lösungen bedeuten aber auch Lösungen, die sich im Verteilungsgleichgewicht befinden, wenn die bezüglichen Lösungsmittel sich nicht mischen.

Dem Verteilungsgleichgewicht im genauen Wortsinne werden die gesättigten Lösungen immerhin nahe stehen. Nun findet man aber durch eine einfache Betrachtung, dass die Beziehung

$$
\frac{D_{1}}{D_{2}}=\sqrt[3]{\frac{C_{1}}{C_{2}}}
$$

$\left(D_{1}\right.$ und $D_{2}$ die Dielektrizitätskonstanten, $C_{1}$ und $C_{2}$ die Ionenkonzentrationen beim Verteilungsgleichgewicht) dann besteht, wenn die Ionen sich nur nach Massgabe ihrer Ladung verteilen, also ohne dass ihre spezifische chemische Natur mit zur Geltung käme4). Die weitere Folge eines solchen Verhaltens wäre, dass in unserer früher angeschriebenen Kette $\pi_{3}$ Null ist, und somit $\pi_{1}=\pi_{2}$.

1) Krouchkoll, Ann. de Chim. et de Phys. (6) 17, 129 (1889).

2) Vgl. H. Freundlich, Kapillarchemie, 2. Aufl., S. 415.

3) P. Walden, Zeitschr. f physik. Chemie 54, 229 (1906); 55, 707 (1906).

4) E. Baur, Zeitschr. f. Elektrochemie 11, 936 (1905); 12, 725 (1906). 
Es war nun sehr bemerkenswert, dass eine Messung der Elektrokapillarkurve in gesättigter wässeriger und gesättigter alkoholischer Lösung von Chlorkalium und Chlorammonium von E. Newbery') in der Tat diese Gleichheit von $\pi_{1}$ und $\pi_{2}$ dartat. Dieser Spur musste man nachgehen. War es nur ein zufälliges Zusammentreffen oder kam ihm allgemeine Geltung zu?

Zunächst ist der Befund von Newbery nur mit Vorbehalt in unserem Sinne zu verwerten, denn die gesättigten rein wässerigen und rein alkoholischen Lösungen stehen unter sich nicht im eigentlichen Sinne im Verteilungsgleichgewicht. Es war nun meine Aufgabe, ein solches Versuchsmaterial zu beschaffen, welches geeignet wäre, die auf Grund der soeben herangezogenen experimentellen Ergebnisse vermuteten Zusammenhänge in helleres Licht zu stellen.

\section{Die Versuchsanordnung.}

Es wurde mit dem gewöhnlichen Kapillarelektrometer nach Lippmann mit senkrechter Kapillare gearbeitet. Die anzulegende Spannung wird von einem kalibrierten Gefällsdraht mit Gleitkontakt abgezweigt, das Arbeitselement durch Kompensation unter Verwendung eines Weston-Normalelementes geeicht. Gemessen wird die Verschiebung des kapillaren Meniskus vor der Okularskala des Beobachtungsmikroskops. Dies setzt voraus, dass die Kapillare in dem für die Messung verwendeten Teile merklich zylindrisch ist. Nach einiger Übung gelingt es meist, kapillare Spitzen von der erforderlichen Feinheit zu ziehen, die nahe der Spitze eine brauchbare zylindrische Strecke haben. Es ist viel bequemer und auch sicherer, die Verschiebung des Meniskus zu messen, als durch Verschieben des Niveaugefässes jene auszugleichen und die Drucksäule zu messen. Der Durchmesser der verwendeten Kapillaren betrug schätzungsweise $50 \mu$, der der feinsten etwa $15 \mu$. Letztere trug in 1 norm. Kaliumnitratlösung eine Quecksilbersäule von etwa $400 \mathrm{~mm}$ Höhe. Sehr viel kommt darauf an, wie die Kapillaren gereinigt und aufbewahrt werden. Nach jeder Messung, speziell einer nichtwässerigen Lösung wurden die Kapillaren in heisses destilliertes Wasser getaucht und durch Heben und Senken des, mit dem Kapillarrohr durch einen Schlauch verbundenen Quecksillberniveaugefässes, das Quecksilber aus der Kapillarspitze zum Austropfen gebracht, bzw. Wasser in die Kapillare eingesogen. Dies wurde mehrmals wiederholt, dann die Spitze in reine Schwefelsäure getaucht und

1) E. Newbery, a. a. 0. 
die Handlung wiederholt, worauf dann die Kapillare wieder tüchtig mit heissem destilliertem Wasser ausgewaschen wurde. Während des Nichtgebrauchs tauchte die Kapillarspitze immer in destilliertes Wasser ein, um das Eindringen von Staub zu verhüten. Auf diese Weise behandelt, konnte eine Kapillare jeweils sehr lange gebraucht werden. Selbst die Abscheidung von Wasserstoff bei zu starker Polarisation schadet nicht, wie auch Paschen ${ }^{1}$ ) bemerkt. Besonderer Wert muss auf die Reinheit des Quecksilbers gelegt werden. Das Reinigen geschah durch Eintropfen in verdünnte Salpetersäure und nachheriges De- . stillieren. Es wird also stets frisch destilliertes Quecksilber verwendet.

Zur Messung taucht die Kapillarspitze in ein kleines, etwa $5 \mathrm{~cm}$ hohes Wägegläschen ein, dessen Boden mit Quecksilber bedeckt ist. Aussen wird ein Deckgläschen aufgeklebt zur besseren Ablesung und Verhütung der Parallaxe. In das mit der zu untersuchenden Lösung gefüllte Gläschen tauchte die Kapillarspitze bis $2 \mathrm{~cm}$ tief hinein. Die Spitze soll möglichst tief eintauchen, weil bei flüchtigen Flüssigkeiten an der Oberfläche durch Verdunsten Konzentrationsunterschiede auftreten können. Die Okularskala umfasste 100 Teilstriche und es konnten die Zehntel noch geschätzt werden. Der Meniskus wurde mit Hilfe der Skala so eingestellt, dass der Abstand desselben von der Kapillarspitze in der Nullage wenigstens für Messungen, die miteinander verglichen werden sollten, immer derselbe blieb. Der Ausschlag des Meniskus wurde erst dann abgelesen, wenn sich zeigte, dass durch eine leichte Erschütterung des Schlauches, welcher das QuecksilberNiveaugefäss mit dem Kapillarrohr verband, der Meniskus frei beweglich war, also das Quecksilber nicht an der Wand hängen blieb. Die Ablesung wurde nur verwertet, wenn der Meniskus nach Aufhebung der Polarisation auf den Nullpunkt, also auf den Ausgangspunkt zurückkehrte, und wurde für jeden Potentialwert mehrmals wiederholt. Die Beweglichkeit des Meniskus war allgemein geringer bei niederen Palarisationsströmen, also auf dem aufsteigenden Ast, wie bei höheren, also auf dem absteigenden Ast. War die Lösung sehr viskos und blieb das Quecksilber an der Wandung der Kapillare kleben, so half oft eine kurze starke negative Polarisation. Vor Beginn der Messung ist das Kapillarelektrometer einige Zeit kurz geschlossen $\mathrm{zu}$ halten, denn es kommt oft vor, dass die Kapillare zu tropfen anfängt, wenn die beiden Elektroden kurz geschlossen werden. Ist das Maximum aus

1) Paschen, Ann. d. Physik 39, 44 (1890). 
einem Grunde irgendwelcher Art schwer zu bestimmen, so kann folgendes Beobachtungsverfahren dienen. Ist die angelegte EK ausgeschaltet, das Kapillarelektrometer aber noch nicht kurz geschlossen, also der Meniskus isoliert, so geht derselbe für Potentialwerte jenseits des Maximums zuerst auf den Maximalausschlag zurück, um dann langsam zum Nullpunkt vorzurücken. Für Kräfte diesseits des Maximums, also für den positiven Ast, kehrt der Ausschlag sofort auf die Nullage zurück. Das negativ geladene Quecksilber wird natürlich, sobald es isoliert ist, der Lösung positive Ionen entziehen, es wird zunächst völlig entladen, die Oberflächenspannung geht auf ihr Maximum, darauf ladet es sich positiv, der Meniskus geht langsam auf den Nullpunkt zurück. Dieses Verhalten, von dem auch Paschen 1) berichtet, konnte ich oft verwerten. Der Hauptwert bei den Messungen wurde auf die Ermittlung der maximalen Oberflächenspannung bzw. des absoluten Potentials gelegt. Es sind daher in der Nähe des Maximums der Elektrokapillarkurve viel mehr Punkte gemessen worden, als die zugehörigen Tabellen jeweils aufweisen. Am zuverlässigsten lässt es sich durch Eingabelung, also Annäherung von Seiten des positiven und negativen Astes bestimmen. Die Fehlergrenze betrug durchschnittlich 10 Millivolt, doch variierte sie natürlich je nach der $\mathrm{zu}$ messenden Phase. Bei wässerigen Lösungen konnte sie auf 5 Millivolt heruntergehen, bei nichtwässerigen in Ausnahmefällen bis 20 Millivolt steigen.

\section{Die Messungen.}

Ich begann damit, die Messungen von Newbery an gesättigten Chlornatriumlösungen in Wasser und in reinem Alkohol zu wiederholen. Sein Befund war unschwer zu bestätigen. Um nun zu prüfen, wie weit es sich um eine Eigenschaft beliebiger, gesättigter Lösungen handelt, dehnte ich die Messungen nach zwei Richtungen aus: 1. auf ein anderes Salz, wozu Kaliumnitrat gewählt wurde; 2. auf Gemische von Alkohol und Wasser. Diese Messungen waren mehr vorbereitender Art und sollten dazu dienen, zu den dann in Angriff genommenen Messungen an Verteilungsgleichgewichten in Kontrast zu treten. Meine Untersuchung zerfällt also in zwei Abschnitte: Der eine handelt von wässerigen und alkoholischen Lösungen, der andere von Paaren unvollständig mischbarer Lösungsphasen.

1) Paschen, Ann. d. Physik 39, 49 (1890). 


\section{Wässerige und alkoholische Lösungen.}

a) Gesättigt an Chlornatrium.

Präparate: Kahlbaumsches Chlornatrium „pro analysi“, wird in Wasser gelöst, mit Alkohol ausgefällt, das abgepresste Kristallmehl im Luftbad getrocknet. - Alkohol, absolut und benzolfrei, dreimal über Ätzkalk destilliert. Wasser, gewöhnlich destilliertes.

Die Lösungsmittel wurden mit dem Salz und einem Zusatz von Mercurochlorid auf der Schüttelmaschine geschüttelt bis zur Sättigung.

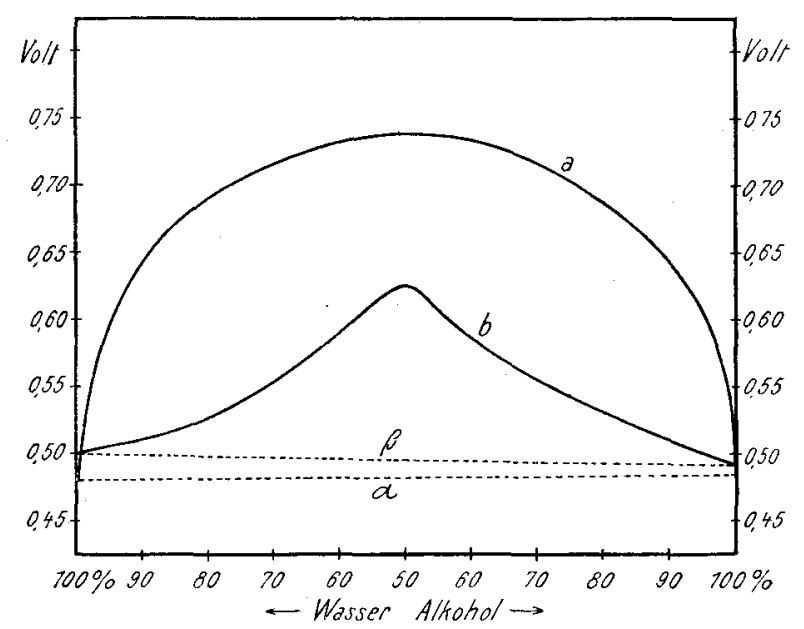

Fig. 1.

Die Messungen sind aufgezeichnet in Tabelle 1a. Die Reproduzierba keit derselben wurde geprüft, doch zur Platzersparnis hier weggelassen. Die Messungen waren leicht auszuführen bei stark wässerigen Lösungen, bei zunehmendem Alkoholgehalt immer schwerer. In der rein alkoholischen Lösung blieb das Quecksilber oft kleben, der Meniskus stellte sich träge ein. Immerhin liess sich das Maximum wohl bestimmen. Die Kurven der alkoholischen Lösungen zeigen gegenüber den mehr wässerigen Kurven eine Abflachung, sonst ist ihr Aussehen das normale. Fig. 1, Kurve $a$, zeigt die Abhängigkeit des Potentials für die maximale Oberflächenspannung von der prozentualen Zusammensetzung von Alkohol und Wasser. Die Prozente bedeuten Volumenprozente von reinem Wasser und Alkohol. Die Gerade $\alpha$ verbindet die absoluten Potentiale für die gesättigten Lösungen der reinen Lösungsmittel. Sie 
Tabelle 1a (hierzu Fig. 1).

Anmerkung: Skt. bedeutet die abgelesenen Skalen-

\begin{tabular}{|c|c|c|c|c|c|c|c|c|c|c|}
\hline \multirow[t]{2}{*}{$\begin{array}{c}\text { Zu- } \\
\text { sammen- } \\
\text { setzung }\end{array}$} & \multicolumn{2}{|c|}{$\begin{array}{l}\text { Wasser } \\
100 \%\end{array}$} & \multicolumn{2}{|c|}{$\begin{array}{c}\text { Alkohol } \\
100 \%\end{array}$} & \multicolumn{2}{|c|}{$\begin{array}{c}\text { Wasser } \\
90 \%\end{array}$} & \multicolumn{2}{|c|}{$\begin{array}{c}\text { Alkohol } \\
90 \%\end{array}$} & \multicolumn{2}{|c|}{$\begin{array}{l}\text { Wasser } \\
80 \%\end{array}$} \\
\hline & Skt. & Volt & Skt. & Volt & Skt. & Volt & Skt. & Volt & Skt. & Volt \\
\hline & 11.7 & 0.201 & $2 \cdot 0$ & 0.204 & $10 \cdot 0$ & 0.201 & 6.0 & 0.201 & 7.3 & 0.200 \\
\hline & 20.0 & 0.296 & $5 \cdot 0$ & 0.300 & 12.5 & 0.296 & 10.0 & 0.396 & 9.0 & 0.294 \\
\hline & 21.5 & 0.396 & 8.5 & 0.440 & $13 \cdot 5$ & 0.396 & 11.0 & 0.494 & 10.0 & 0.394 \\
\hline & 22.2 & 0.475 & 9.0 & 0.502 & $14 \cdot 8$ & $0: 475$ & 12.0 & 0.592 & 11.0 & 0.492 \\
\hline & 21.0 & 0.592 & 6.0 & 0.600 & $15 \cdot 6$ & 0.592 & 12.5 & 0.650 & 11.5 & 0.588 \\
\hline & $19 \cdot 0$ & 0.690 & 4.0 & 0.700 & 16.0 & 0.670 & 11.0 & 0.709 & 11.8 & 0.666 \\
\hline & 16.2 & 0.788 & $2 \cdot 0$ & 0.800 & 15.5 & 0.748 & 7.0 & 0.788 & 11.5 & 0.705 \\
\hline & 11.2 & 0.885 & 1.5 & 0.896 & 13.8 & 0.885 & 1.0 & 0.885 & 11.6 & 0.784 \\
\hline & 3.5 & 0.981 & 1.0 & 0.996 & & & & & 10.2 & 0.880 \\
\hline & & & & & & & & & 5.2 & 0.978 \\
\hline Maximum & \multicolumn{2}{|c|}{0.480} & \multicolumn{2}{|c|}{0.485} & \multicolumn{2}{|c|}{$0 \cdot 640$} & \multicolumn{2}{|c|}{0.650} & \multicolumn{2}{|c|}{0.690} \\
\hline
\end{tabular}

Tabelle $1 \mathrm{~b}$.

\begin{tabular}{|c|c|c|c|c|c|c|c|c|c|c|}
\hline \multirow[t]{2}{*}{$\begin{array}{c}\text { Zu- } \\
\text { sammen- } \\
\text { setzung }\end{array}$} & \multicolumn{2}{|c|}{$\begin{array}{l}\text { Wasser } \\
100 \%\end{array}$} & \multicolumn{2}{|c|}{$\begin{array}{l}\text { Alkohol } \\
100 \%\end{array}$} & \multicolumn{2}{|c|}{$\begin{array}{c}\text { Wasser } \\
90 \%\end{array}$} & \multicolumn{2}{|c|}{$\begin{array}{l}\text { Alkohol } \\
90 \%\end{array}$} & \multicolumn{2}{|c|}{$\begin{array}{c}\text { Wasser } \\
80 \%\end{array}$} \\
\hline & Skt. & Volt & Skt. & Volt & Skt. & Volt & Skt. & Volt & Skt. & Volt \\
\hline & 10.5 & 0.209 & 2.5 & 0.209 & 12.8 & 0.209 & $9 \cdot 3$ & 0.209 & 14.0 & 0.209 \\
\hline & 10.8 & 0.310 & 2.7 & 0.310 & 17.5 & 0.310 & 13.0 & 0.310 & 17.0 & 0.310 \\
\hline & $11 \cdot 0$ & 0.411 & $3 \cdot 1$ & 0.411 & 17.7 & 0.411 & 13.4 & 0.411 & 17.5 & 0.411 \\
\hline & 11.3 & 0.512 & $4 \cdot 3$ & 0.490 & 17.9 & 0.512 & 13.8 & 0.512 & 18.0 & 0.532 \\
\hline & 10.9 & 0.614 & $4 \cdot 1$ & 0.614 & 17.5 & 0.614 & 13.0 & 0.614 & 17.0 & 0.614 \\
\hline & 9.7 & 0.715 & 3.7 & 0.715 & 14.8 & 0.715 & 11.7 & 0.715 & 16.2 & 0.715 \\
\hline & 7.8 & 0.817 & 3.2 & 0.817 & 11.5 & 0.817 & 10.5 & 0.817 & 14.0 & 0.817 \\
\hline & $5 \cdot 4$ & 0.918 & 2.0 & 0.918 & 8.0 & 0.918 & 8.0 & 0.918 & 7.0 & 0.918 \\
\hline Maximum & & \multicolumn{2}{|c|}{0.492} & \multicolumn{2}{|c|}{0.510} & \multicolumn{2}{|c|}{0.512} & \multicolumn{2}{|c|}{0.525} \\
\hline
\end{tabular}


Tabelle 1a (hierzu Fig. 1).

teile, Volt die zugehörigen Potentialwerte.

\begin{tabular}{|c|c|c|c|c|c|c|c|c|c|c|c|}
\hline \multicolumn{2}{|c|}{$\begin{array}{c}\text { Alkohol } \\
80 \%\end{array}$} & \multicolumn{2}{|c|}{$\begin{array}{c}\text { Wasser } \\
70 \%\end{array}$} & \multicolumn{2}{|c|}{$\begin{array}{c}\text { Alkohol } \\
70 \%\end{array}$} & \multicolumn{2}{|c|}{$\begin{array}{c}\text { Wasser } \\
60 \%\end{array}$} & \multicolumn{2}{|c|}{$\begin{array}{c}\text { Alkohol } \\
60 \%\end{array}$} & \multicolumn{2}{|c|}{$\begin{array}{c}\text { Wasser } \\
\text { (Alkohol) } \\
50 \%\end{array}$} \\
\hline Skt. & Volt & Skt. & Volt & Skt. & Volt & Skt. & Volt & Skt. & Volt & Skt. & Volt \\
\hline 7.0 & 0.200 & 7.0 & 0.200 & 6.5 & 0.200 & 6.0 & 0.200 & 6.5 & 0.200 & $5 \cdot 0$ & 0.102 \\
\hline 8.5 & 0.294 & $8 \cdot 3$ & 0.294 & 7.5 & 0.294 & 7.2 & 0.294 & $7 \cdot 7$ & 0.294 & 8.5 & 0.204 \\
\hline 10.0 & 0.394 & 9.2 & 0.394 & 8.2 & 0.394 & 8.0 & 0.394 & $9 \cdot 3$ & 0.394 & 10.5 & 0.300 \\
\hline 11.3 & 0.492 & 10.0 & 0.492 & 8.8 & 0.492 & 8.7 & 0.492 & 10.0 & 0.492 & $12 \cdot 0$ & 0.402 \\
\hline 12.2 & 0.588 & 10.5 & 0.588 & $9 \cdot 4$ & 0.588 & 9.3 & 0.588 & 10.5 & 0.588 & 13.0 & 0.502 \\
\hline 12.4 & 0.666 & 10.9 & 0.686 & 9.8 & 0.686 & 9.5 & 0.686 & 11.0 & 0.686 & 14.0 & 0.600 \\
\hline 12.0 & 0.784 & $11 \cdot 0$ & 0.705 & 9.8 & 0.784 & 9.3 & 0.784 & 10.8 & 0.784 & 14.5 & 0.710 \\
\hline 9.6 & 0.880 & 10.9 & 0.745 & 7.2 & 0.880 & 8.0 & 0.880 & 8.2 & 0.880 & $14 \cdot 3$ & 0.800 \\
\hline 4.3 & 0.978 & 10.7 & 0.784 & 4.0 & 0.978 & $3 \cdot 2$ & 0.978 & 4.0 & 0.978 & 13.0 & 0.890 \\
\hline & & 9.3 & 0.880 & & & & & & & 11.0 & 0.996 \\
\hline & & 5.85 & 0.978 & & & & & & & & \\
\hline \multicolumn{2}{|c|}{0.690} & \multicolumn{2}{|c|}{0.715} & \multicolumn{2}{|c|}{0.715} & \multicolumn{2}{|c|}{0.735} & \multicolumn{2}{|c|}{0.740} & \multicolumn{2}{|c|}{$0.742 \mathrm{~V}$} \\
\hline
\end{tabular}

Tabelle $1 \mathrm{~b}$.

\begin{tabular}{|c|c|c|c|c|c|c|c|c|c|c|c|}
\hline \multicolumn{2}{|c|}{$\begin{array}{c}\text { Alkohol } \\
80 \%\end{array}$} & \multicolumn{2}{|c|}{$\begin{array}{c}\text { Wasser } \\
75 \%\end{array}$} & \multicolumn{2}{|c|}{$\begin{array}{c}\text { Alkohol } \\
75 \%\end{array}$} & \multicolumn{2}{|c|}{$\begin{array}{c}\text { Wasser } \\
60 \%\end{array}$} & \multicolumn{2}{|c|}{$\begin{array}{c}\text { Alkohol } \\
60 \%\end{array}$} & \multicolumn{2}{|c|}{$\begin{array}{c}\text { Wasser } \\
\text { (Alkohol) } \\
50 \%\end{array}$} \\
\hline Skt. & Volt & Skt. & Volt & Skt. & Volt & Skt. & Volt & Skt. & Volt & Skt. & Volt \\
\hline 6.7 & 0.209 & 13.0 & 0.209 & 10.5 & 0.209 & $6 \cdot 0$ & 0.208 & 9.0 & 0.209 & 9.2 & 0.209 \\
\hline 8.0 & 0.310 & $16 \cdot 0$ & 0.310 & 12.5 & 0.310 & 8.0 & 0.315 & 10.5 & 0.310 & 11.5 & 0.810 \\
\hline 8.4 & 0.411 & 16.8 & 0.411 & 14.0 & 0.411 & 8.6 & 0.410 & 11.2 & 0.410 & 11.9 & 0.411 \\
\hline 8.8 & 0.532 & $17 \cdot 1$ & 0.0532 & 14.5 & 0.551 & 9.5 & 0.510 & 12.1 & 0.592 & $12 \cdot 2$ & 0.512 \\
\hline 8.5 & 0.614 & 16.5 & 0.614 & 12.8 & 0.614 & 9.9 & 0.592 & 11.5 & 0.715 & 12.5 & 0.634 \\
\hline 7.3 & 0.715 & 14.5 & 0.715 & 11.5 & 0.715 & 8.5 & 0.713 & $10 \cdot 0$ & 0.814 & $12 \cdot 0$ & 0.715 \\
\hline $5 \cdot 6$ & 0.817 & 11.2 & 0.817 & 10.0 & 0.817 & 6.0 & 0.814 & $6 \cdot 5$ & 0.915 & 11.0 & 0.817 \\
\hline $3 \cdot 0$ & 0.918 & 7.0 & 0.918 & 6.0 & 0.918 & 3.6 & 0.915 & & & 7.0 & 0.918 \\
\hline \multicolumn{2}{|c|}{0.522} & \multicolumn{2}{|c|}{0.545} & \multicolumn{2}{|c|}{0.551} & \multicolumn{2}{|c|}{0.590} & \multicolumn{2}{|c|}{0.585} & \multicolumn{2}{|c|}{$0.625 \mathrm{~V}$} \\
\hline
\end{tabular}


ist merklich parallel zur Abszisse, entsprechend der Gleichheit der beiden Potentialwerte. Für reinen Alkohol mass ich 0.485 Volt; für reines Wasser 0.480 Volt; während Newbery angibt: für Alkohol 0.483 Volt; für Wasser 0.481 Volt.

Es ergibt sich, dass die Gleichheit der absoluten Potentiale in den rein gesättigten Lösungen sofort verschwindet, wenn gemischt gesättigte an ihre Stelle treten. Dieses lehrt uns, dass nicht etwa jedes beliebige Lösungsmittelgemisch bei Sättigung an einem bestimmten Salz dasselbe absolute Potential erzeugen muss, und dass, wenn wir derartiges später für Phasen finden, die im Verteilungsgleichgewicht stehen, hierin ein charakteristisches Verhalten liegt. Dass die Kurve $a$ auf Fig. 1 scheitelsymmetrisch ist, muss als zufällig gelten. Würde man als Abszisse Gewichtsprozente wählen, statt (unkorrigierte) Volumenprozente, so würde schon eine gewisse Dissymetrie entstehen.

b) Gesättigt an Kaliumnitrat.

Präparate: Käufliches Kaliumnitrat „pro analysi“, behandelt wie Chlornatrium unter a). - Alkohol wie oben.

Die Lösungsmittel werden mit dem Salz geschüttelt, doch ohne Zusatz von Mercuronitrat, denn nach Krüger und Krumreich $\left.{ }^{1}\right)$ kann man auch ohne Mercurosalz bis auf $2 \%$ übereinstimmende Potentialwerte erhalten, indem sich eine ausreichend definierte Mercuroionenkonzentration in der Nachbarschaft der Quecksilberkuppe schon von selbst herstellt. Die Messresultate finden sich in Tabelle $1 \mathrm{~b}$, die Abhängigkeit des absoluten Potentials von der prozentischen Zusammensetzung des Alkoholwassergemisches ist in Fig. 1 Kurve $b$ abgebildet. Das Verhalten der beiden reingesättigten Lösungen ist dem der Chlornatriumlösungen recht ähnlich; immerhin sieht man an der schwachen Neigung der Geraden $\beta$ auf Fig. 1, dass die beiden Potentialwerte doch merklich ungleich sind - 0.500 Volt gegen 0.492 Volt - ein Unterschied, der die Fehlergrenze der Maximumbestimmung schon übersteigen dürfte. Dies zum Zeichen, dass die Gleichheit der Potentialwerte der rein gesättigten Lösungen, wie beim Chlornatrium, doch wohl mehr zufälligen Charakter hat. Die beiden Lösungen sind ja nicht im strengen Sinne solche, die im Verteilungsgleichgewicht stehen. Die Mischungskurve ist wieder scheitelsymmetrisch, vgl. die bezügliche Bemerkung beim Chlornatrium.

1) Krüger und Krumreich, Zeitschr. f. Elektrochemie 19, 619 (1913). 


\section{Paare unvollständig mischbarer Iösungsphasen.}

Der eine Partner ist stets eine wässerige Phase. Der andere wurde unter den zahlreichen organischen Lösungsmitteln ausgewählt. Als einschränkend bei der Auswahl tritt die Bedingung auf, dass der organische Partner eine gewisse Wasser- und damit dann auch eine gewisse Salzlöslichkeit habe, da sonst die nichtwässerige Phase zu schlecht leiten würde. Die Auswahl war natürlich auch dadurch eingeschränkt, weil viele organische mit Wasser nicht mischbare Stoffe, sei es schon von selbst oder in Berührung mit Wasser Veränderungen unterworfen sind (Aldehyde, Säurechloride usw.). So konnte z. B. Benzaldehyd nicht verwendet werden, wegen der raschen Bildung von Benzoesäure.

Es wurden mit folgenden nichtwässerigen Phasen Messungen gemacht:

$\begin{array}{ll}\text { Amylalkohol } & \text { Anilin } \\ \text { Phenol } & \text { Chloroform } \\ \text { Furfurol } & \text { Propylalkohol } \\ \text { Essigsäureäthylester } & \text { Isobuthylalkohol } \\ & \text { Äther. }\end{array}$

Auf die Reinheit der Stoffe ist sehr zu achten. Sämtliche Lösungsmittel wurden daher vor Verwendung frisch destilliert und nur eine Fraktion nahe dem Siedepunkt des reinen Stoffes verwendet. Sind in der Lösung Säurespuren vorhanden, herrührend von der Herstellung, z. B. bei Essigäther und Äther, so müssen dieselben durch Behandeln mit Soda entfernt werden.

Es zeigte sich nun, dass man zwar mit Phenol, Furfurol und Propylalkohol im Verteilungsgleichgewicht mit Wasser gute Elektrokapillarkurven erhalten konnte, nicht aber mit den anderen nichtwässerigen Phasen, sei es, dass die Löslichkeit des Salzes zu gering war, sei es der zu grossen Viskoșität halber. Die Ausschläge waren nicht scharf genug, der Meniskus ging nicht mehr auf den Nullpunkt zurück, die Kurve war nicht reproduzierbar, oder es wurden überhaupt keine Ausschläge beobachtet. Dieser Übelstand konnte nun aber beseitigt werden, indem ich ternäre Gemische herstellte, durch Zusatz einer passenden Menge Äthylalkohol zum System.

Als Elektrolyten wurden Kaliumchlorid, Kaliumnitrat und Mercuronitrat verwendet. Die Lösungsmittel wurden mit dem betreffenden Salze auf der Schüttelmaschine bis zur Sättigung geschüttelt. Bei den Versuchen mit Kaliumchlorid wurde immer etwas Mercurochlorid 
beigesetzt. Bei Kaliumnitrat wurde Mercurosalz weggelassen aus schon angeführten Gründen.

Die Versuche mit Mercuronitrat allein schlugen zunächst fehl. Wegen zu weiter Kapillaren pendelte der Meniskus bei Polarisation in der Kapillare hin und her. Bekanntlich fand Paschen ${ }^{1}$ ) in einer $3 \mathrm{~mm}$ weiten Kapillare Quecksilber gegen saure Mercuronitratlösung völlig unpolarisierbar. Später konnten aber Krüger und Krumreich ${ }^{2)}$ mit genügend feiner Kapillare die Diffusion der Mercuroionen soweit zurückdrängen, dass es ihnen gelang, selbst mit normaler Kaliumnitratlösung, die in bezug auf Mercuronitrat 0.1 norm. war, Elektrokapillarkurven aufzunehmen. Ich versuchte nun auch die Kapillaren zu verfeinern, und konnte schliesslich mit einer solchen, die ungefähr $13 \mu$ Durchmesser hatte, die Kurven mit:

und

0.1 norm. $\mathrm{Hg}_{2}\left(\mathrm{NO}_{3}\right)_{2}+1$ norm. $\mathrm{KNO}_{3}+0.1$ norm. $\mathrm{HNO}_{3}$

$$
0.01 \quad \mathrm{Hg}_{2}\left(\mathrm{NO}_{3}\right)_{2}+1 \quad, \quad \mathrm{KNO}_{3}+0.1 \quad, \quad H N O_{3}
$$

ebenfalls aufnehmen. Mit diesen beiden Lösungen wurden dann auch die nichtwässerigen Phasen geschüttelt und mit Erfolg elektrometrisch gemessen. Der Zusatz der Säure hilft dazu, die Hydrolyse des Mercurosalzes zurückzudrängen. Da das käufliche Mercuronitrat immer Mercurinitrat enthält, so wurde beim Schütteln mit obigen Lösungen noch ein Tropfen Quecksilber zugegeben, um das Mercurisalz zu reduzieren. Mercuronitrat setzt die Oberflächenspannung des Quecksilbers stark herab; z. B. trug die Kapillare in einer 1 norm. Lösung von Kaliumnitrat etwa $400 \mathrm{~mm}$ Quecksilbersäule, dagegen in einer 0.1 norm. Mercuronitratlösung nur etwa $300 \mathrm{~mm}^{3}$ ).

Es folgen nun die Messungen in Gestalt von Tabellen und Kurven. Die ersteren müssen wiedergegeben werden, um die Ermittlung der Maxima nachprüfen zu können, die letzteren, um den Überblick zu ermöglichen. Die in den Tabellen in der Spalte „Skt." eingeklammert angeschriebene \pm Zahl bedeutet eine willkürliche Verrückung der Ordinate um diesen Betrag, um die betreffenden Kurven übereinander und übersichtlich zeichnen zu können; (:2) (Tabelle 1a und $3 \mathrm{~b}$ ) bedeutet: abgelesene Skalenteile dividiert durch 2. Die Potentialwerte der leergelassenen bzw. punktierten Voltkolonnen sind identisch mit denjenigen in der vorherigen ausgefüllten Voltkolonne.

1) Paschen, Ann. d. Physik 39, 55 (1890).

2) Krüger und Krumreich, Zeitschr. f. Elektrochemie 19, 617 (1913).

3) Vgl. Paschen, Ann. d. Physik 39, 56 (1890). 
a) Amylalkohol norm. (Fig. 2 und 2a).

Vorauszuschicken sind einige Bemerkungen, die sich auf das Verhalten der Phasen beziehen. Man erhält Kurven nur unter Zusatz von

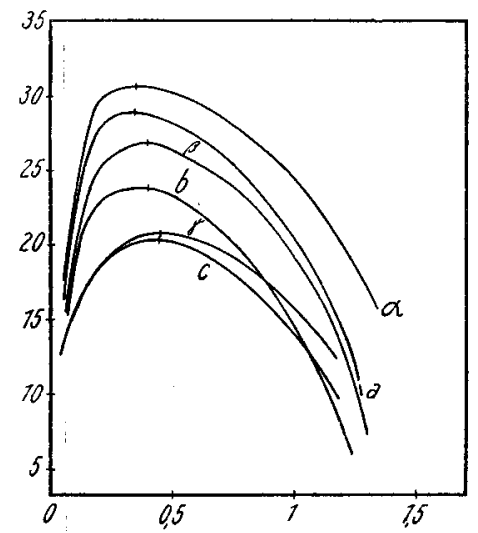

Fig. 2.

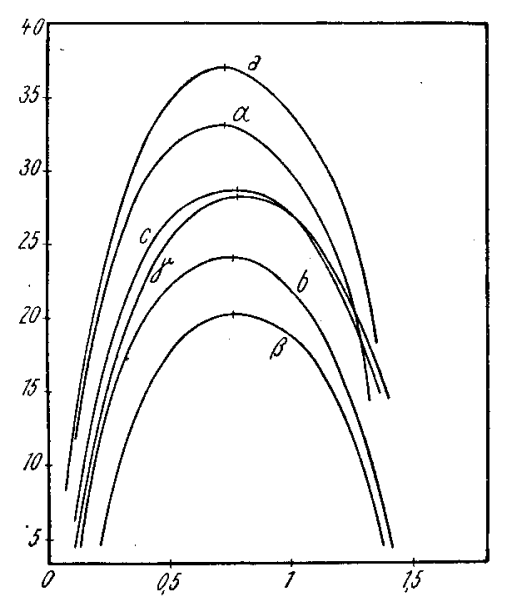

Fig. 3.

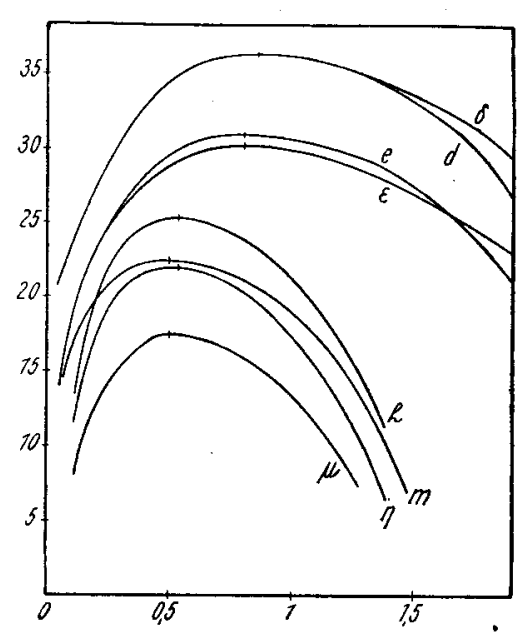

Fig. 2 a.

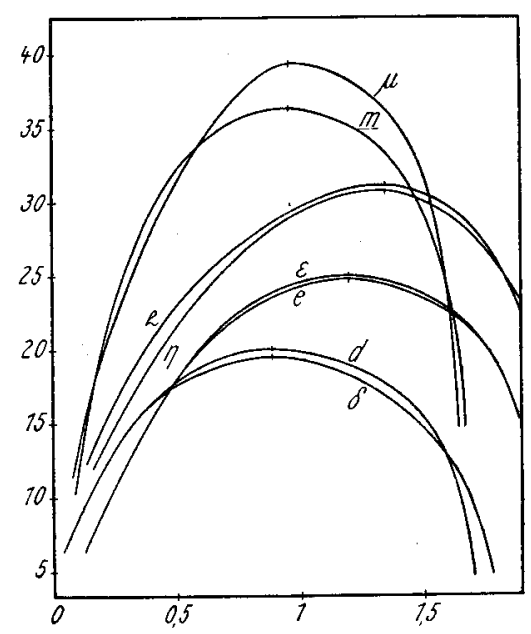

Fig. 3 a.

Äthylalkohol. Die Herstellung der Phasen ist aus den Tabellen 2 und 2a ersichtlich. Der ansteigende Kurvenast ist namentlich mit Mercuronitrat (Fig. 2a: $d, \delta, e, \varepsilon$ ) bedeutend steiler als der abfallende. Hingewiesen sei auch auf die Verschiebung des absoluten Potentials gegen- 
über den Kurven mit Kaliumnitrat nach der Seite höher negativer Werte um 0.3 Volt. Dieser Einfluss des Mercuroions wird später immer wieder und in derselben Grössenordnung gefunden. , Er steht im Einklang mit der Formel für die elektrolytischen Potentiale nach Nernst.

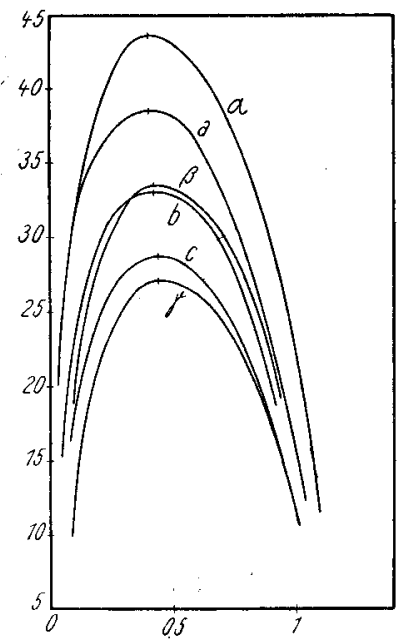

Fig. 4.

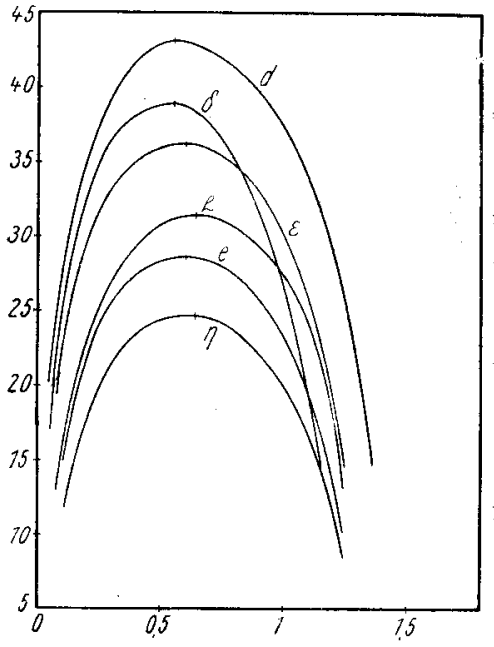

Fig. 4 a.

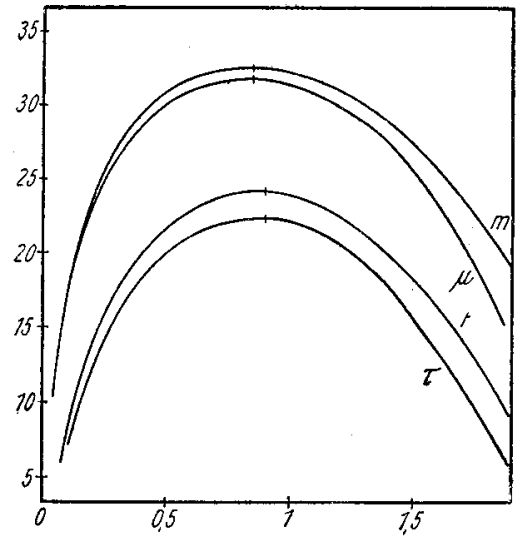

Fig. $4 \mathrm{~b}$.

b) Phenol (vgl. Fig. 3 und 3a, S. 15).

Phenol mit Wasser und Kaliumchlorid geschüttelt, ergaben für beide Phasen sehr schöne parabelförmige Kurven (Fig. 3). ' Der Konzentrationseinfluss ist deutlich; er beträgt für einen Konzentrationsfall 
von 1 norm. auf 0.5 norm. 20 Millivolt, nahe übereinstimmend mit der Theorie. Phenol mit 0.1 norm. Kaliumchlorid geschüttelt, lieferte nur den aufsteigenden Ast der Kurve, der absteigende Ast war nicht zu erhalten, die Kurve verlief parallel der Abszisse. Gouy ${ }^{1}$, der diese Erscheinung diskutiert, führt sie auf eine Wasserstoff belegung der Quecksilberkuppe zurück. - Die Kurven mit Kaliumnitrat zeigen einen bedeutend steileren Aufstieg wie Abfall.

c) Furfurol (vgl. Fig. 4, 4 a und 4b, S. 16).

Die Messungen konnten ohne Alkoholzusatz gemacht werden. Die Kurven sind durchweg schön parabelförmig, der Konzentrationseinfluss deutlich. Mit 0.1 norm. Kaliumchlorid geschütteltes Furfurol zeigte ein gleiches Verhalten wie Phenol.

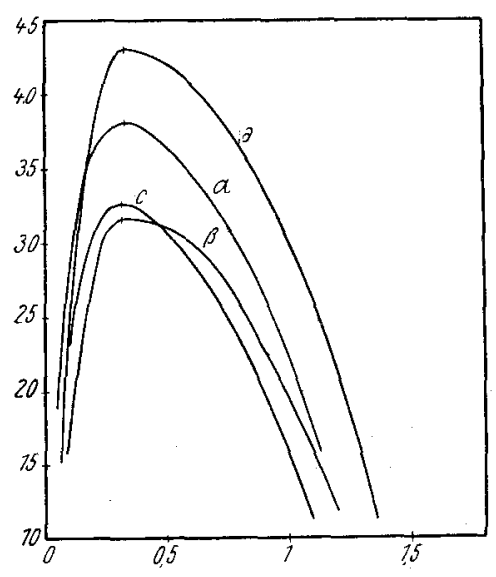

Fig. 5.

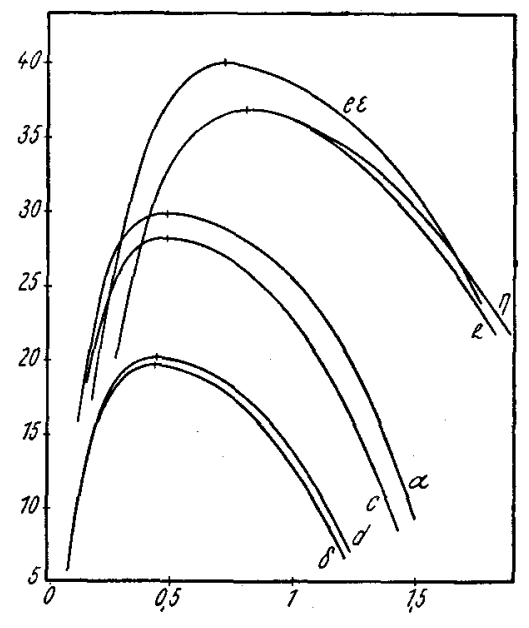

Fig. 5 a.

d) Essigsäureäthylester (vgl. Fig. 5 und 5a).

Mit Essigsäureäthylester konnten ohne Alkoholzusatz keine Kurven aufgenommen werden; es kam überhaupt kein Ausschlag zustande, wahrscheinlich wegen der allzu geringen Löslichkeit der betreffenden Salze in Essigäther. Mit Kaliumchlorid sind die Kurven sehr unsymmetrisch, mit Kaliumnitrat sind sie mehr gerundet und symmetrischer, diejenigen von Mercuronitrat zeigen das Maximum wieder stark nach rechts verschoben.

1) Gouy, Ann. d. Chim. et de Phys. (7) 29, 145 (1903). 
e) Äther (vgl. Fig. 6 und 6a).

Äther zeigte bei den Messungen ein ganz ähnliches Verhalten wie Essigester. Auch mit Alkoholzusatz bieten die Messungen. Schwierigkeiten. Die Kurven sind stark unsymmetrisch. Die scharfe Biegung links vom Maximum auf dem positiven Ast ruft fast den Eindruck eines Knickes hervor.

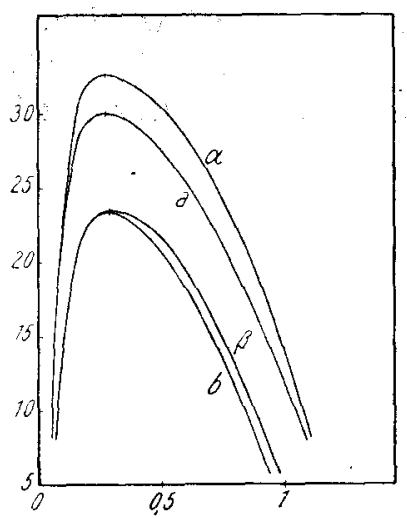

Fig. 6.

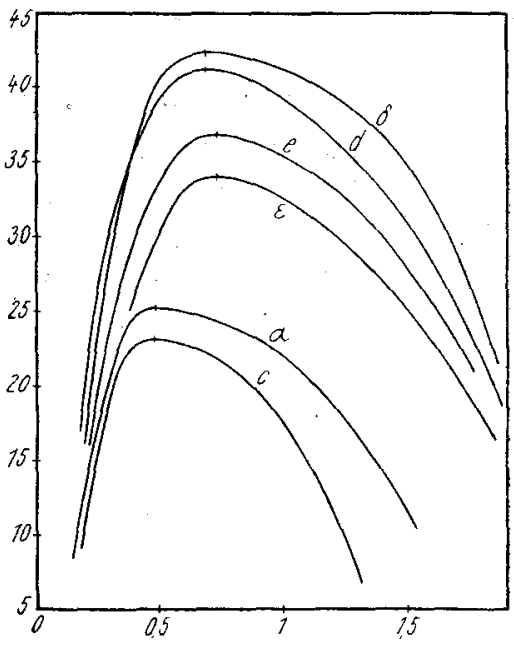

Fig. 6 a.

f) Anilin (vgl. Fig. 7 und 7a, S. 19).

Bei Anilin, mit Wasser und Kaliumchlorid geschüttelt, konnten keine Ausschläge erzeugt werden, wohl wegen der zu geringen Leitfähigkeit, denn der Quecksilbermeniskus war sehr leicht beweglich in der Kapillare. Mit Kaliumnitrat erhielt man den aufsteigenden Ast der Elektrokapillarkurve, der dann parallel der Voltaxe sich verlängerte. Bei Zusatz von Alkohol waren die Lösungen messbar. Mit Kaliumchlorid wurden recht unsymmetrische Kurven gefunden, diejenigen von Kaliumnitrat waren im Gegensatz dazu sehr schön symmetrisch und parabelförmig. Mit saurer Mercuronitratlösung geschüttelt (Fig. 7a: $e, \varepsilon$ ) zeigte sich eine bemerkenswerte Verschiebung des Maximums der wässerigen Phase gegenüber der nichtwässerigen nach der Seite des kleineren negativen Potentials. Die Säure bedingt das Auftreten von positiven Aniliniumionen in der wässerigen Phase, diese werden auf dem negativen Aste adsorbiert, das Maximum wird nach der Seite des positiven Astes hin verschoben ${ }^{1}$. Wir ersehen daraus, dass in den

1) Vgl. H. Freundlich, Kapillarohemie, 2. Aufl., 1922, S. 404. 
normalen Fällen, wo die Maxima entsprechender Kurven zusammenfallen, Trübungen der Verhältnisse durch Ionenadsorption nicht bestanden haben, und dass andererseits, wenn solche Störungen vorliegen, sie auch zutage treten.

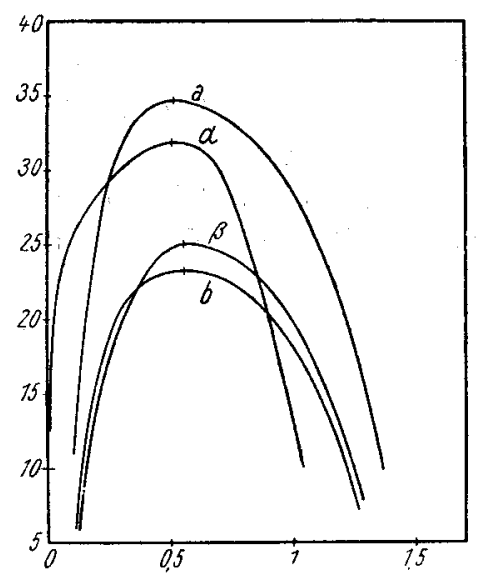

Fig. 7.

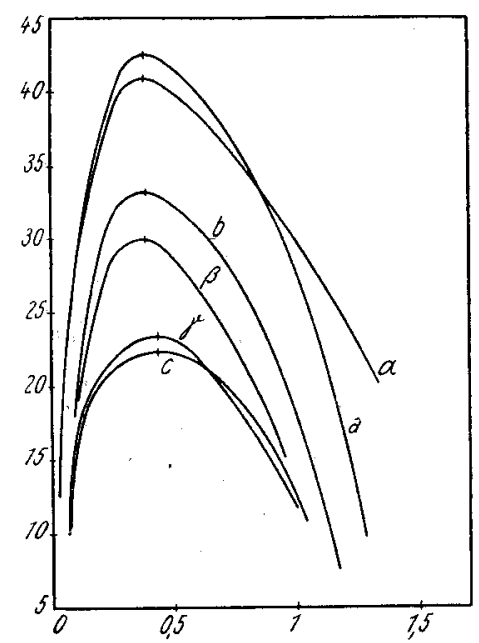

Fig. 8.

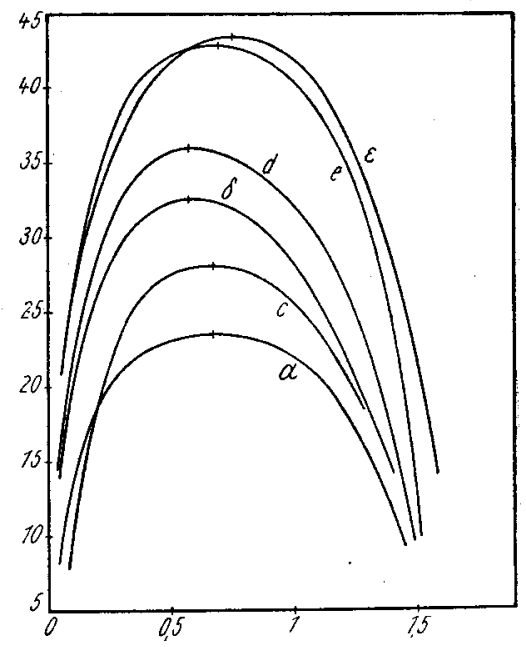

Fig. 7 a.

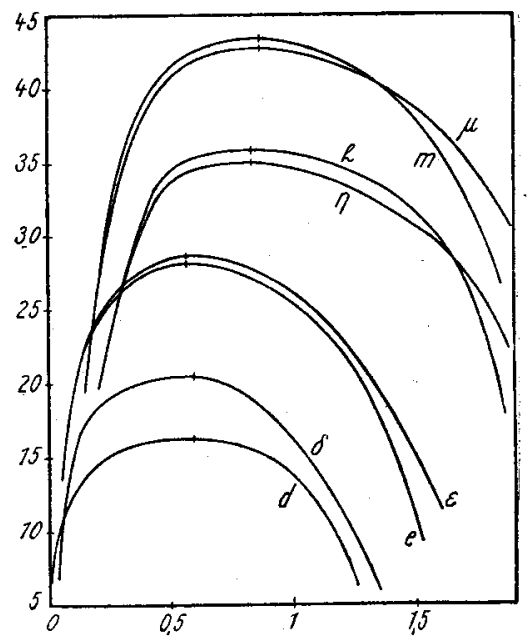

Fig. 8 a.

g) Chloroform (Fig. 8 und 8a).

Die Kurven (mit Alkoholzusatz erhalten) zeigen sowohl für Kaliumnitrat wie für Kaliumchlorid symmetrische Formen. Die Kurve von 0.01 norm. Mercuronitrat ist stark abgeflacht (Fig. 8a: $h, \eta$ ), das Maximum schwer zu bestimmen. 
h) Propylalkohol (vgl. Fig. 9).

Hier liegt insofern ein besonderer Fall vor, als Propylalkohol sich mit Wasser vollkommen mischt, und eine gewisse Salzkonzentration angewendet werden muss, um eine Phasentrennung zu erzielen, $d . h$. den untern kritischen Entmischungspunkt unter die Zimmertemperatur herabzudrücken. Nach V. Rothmund 1) wird bei einem Gemisch von $39.1 \%$ Propylalkohol in Wasser, das in bezug auf Kaliumchlorid 1.0181 norm. ist, die untere kritische Entmischungstemperatur auf $8,4^{\circ}$ heruntergedrückt. Ich erhielt z. B. mit einem Gemisch (siehe Tabelle 9) von $20 \mathrm{ccm}$ Propylalkohol und $30 \mathrm{ccm}$ Wasser, nach Sättigung desselben mit Kaliumchlorid bei Zimmertemperatur $\left(18^{\circ}\right)$, gerade die Ent-

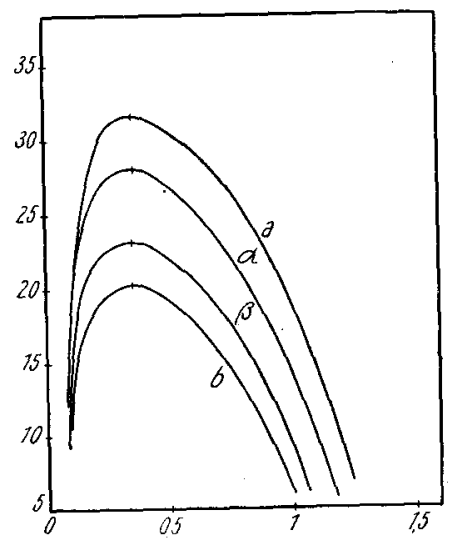

Fig. 9.

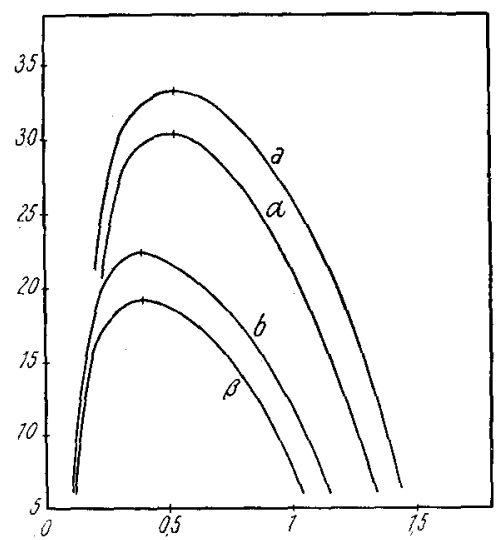

Fig. 10.

mischung, desgleichen mit einem Teil Propylalkohol und einem Teil 2 norm. Kaliumchloridlösung. Die erhaltenen Kurven der entmischten Phasen zeigen normales Verhalten. Der Aufstieg ist bedeutend steiler wie der Abfall. Es ist ein Konzentrationseinfluss vorhanden beim Übergang von gesättigten Lösungen zu nichtgesättigten.

\section{i) Is obutylalkohol (vgl. Fig. 10).}

Ohne Alkoholzusatz wurde bei der nichtwässerigen Phase eine Kurve mit aufsteigendem und zur Abszisse parallelen Ast erhalten. Bei Alkoholzusatz zeigen die erhaltenen Kurven der beiden Phasen ungefähr denselben Verlauf wie diejenigen von Propylalkohol.

1) V. Rothmund, Löslichkeit und Löslichkeitsbeeinflussung (Handb. d. angew. phys. Chem. VII), S. 161, Barth, Leipzig 1907. 


\section{Anhang.}

Auf Fig. 11 sind zum Vergleich die Kurven von saurer 0.1 norm. und 0.1 norm. rein wässeriger Mercuronitratlösung, bevor sie mit den verschiedenen organischen Phasen in Verteilung gesetzt wurden, ferner die Kurven von 1 norm. Kaliumnitrat und 0.01 norm. rein wässeriger Kaliumnitratlösung wiedergegeben. Die Potentialverschiebung in bezug auf die Mercuroionenkonzentration ergab sich in richtiger Grösse und Vorzeichen. Übrigens stehen die Werte für das Maximum in völliger Übereinstimmung mit denen von F. Krüger und Krumreich. Der Vergleich der Maximumwerte in rein wässeriger Lösung zu denen in den gemischt-wässerigen Lösungen ergibt die Veränderung, die der organische Nichtelektrolyt zugleich im elektrolytischen Lösungsdruck des Metalles und in der Konzentration seiner Ionen hervorruft, beides als Folge der geänderten Dielektrizitätskonstante des Mediums. Ist $\pi_{3}$ $\mathrm{zu}$ vernachlässigen, so kann man

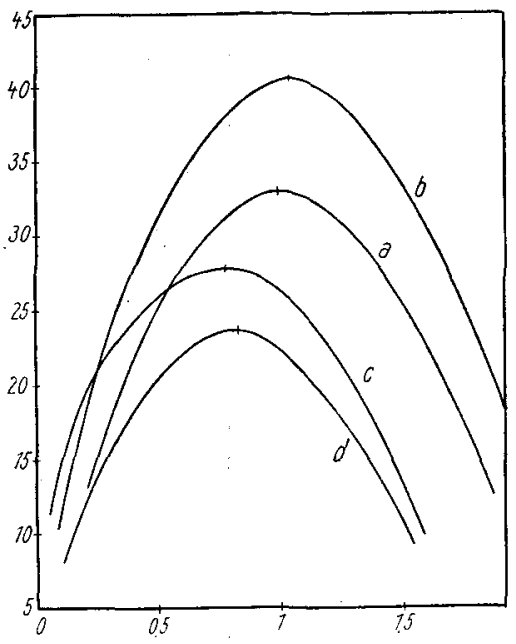

Fig. 11. folgern, dass die elektrolytischen Lösungstensionen $\pi_{1}$ und $\pi_{2}$ eines Metalles in den Medien mit den Dielektrizitätskonstanten $D_{1}$ und $D_{2}$ sich verhalten wie:

$$
\left.\frac{\pi_{1}}{\pi_{2}}=\left(\frac{D_{1}}{D_{2}}\right)^{3} 1\right)
$$

Die Änderungen, die $\pi_{1}$ durch den Zusatz des organischen Nichtelektrolyten und $\pi_{2}$ durch den Zusatz des Wassers erleiden, und die schliesslich - wie die Messungen zeigen werden - zur Gleichheit von $\pi_{1}$ und $\pi_{2}$ führen, scheinen hierdurch genügend erklärt $\mathrm{zu}$ sein, ohne dass es nötig wäre, eine besondere "elektrotonische“ Wirkung des organischen Nichtelektrolyten einzuführen, wie H. Freundlich ${ }^{2}$ ) in seiner Kapillarchemie versucht.

1) E. Baur, Zeitschr. f. Elektrochemie 12, 726 (1906).

2) H. Freundlich, 2. Aufl., S. 401. 


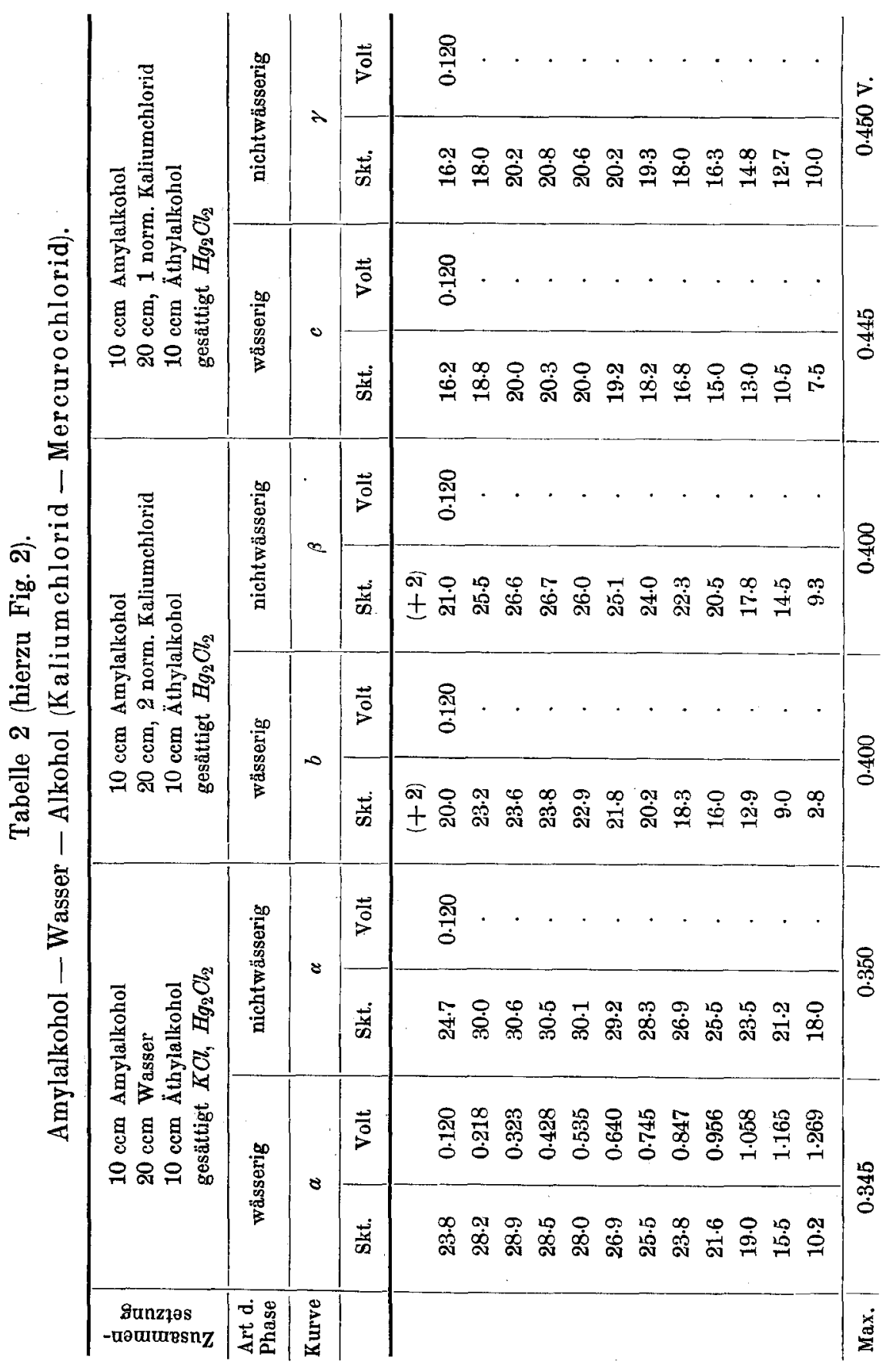


Elektrokapillarkurven in nichtwässerigen Lösungen.

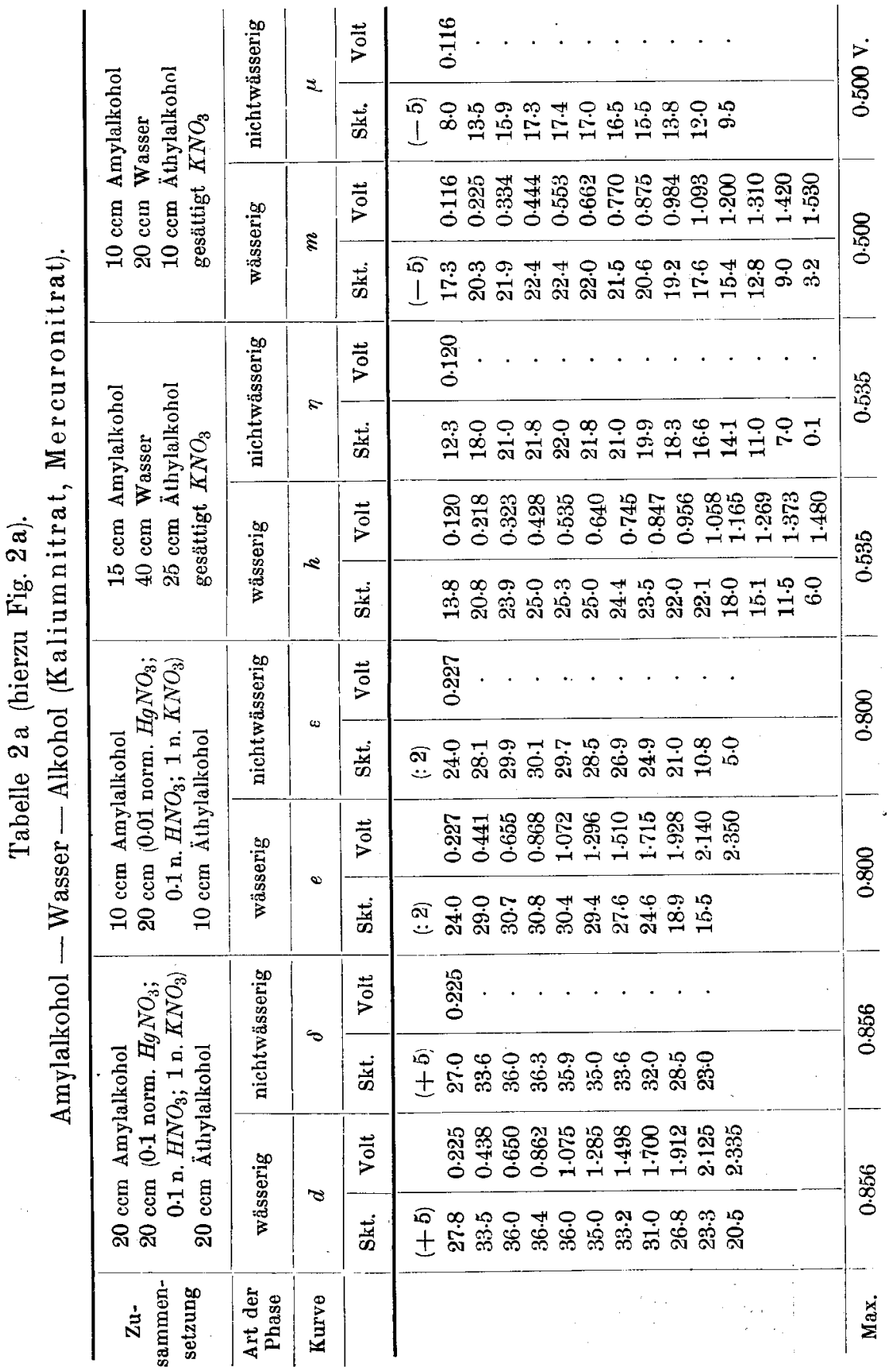




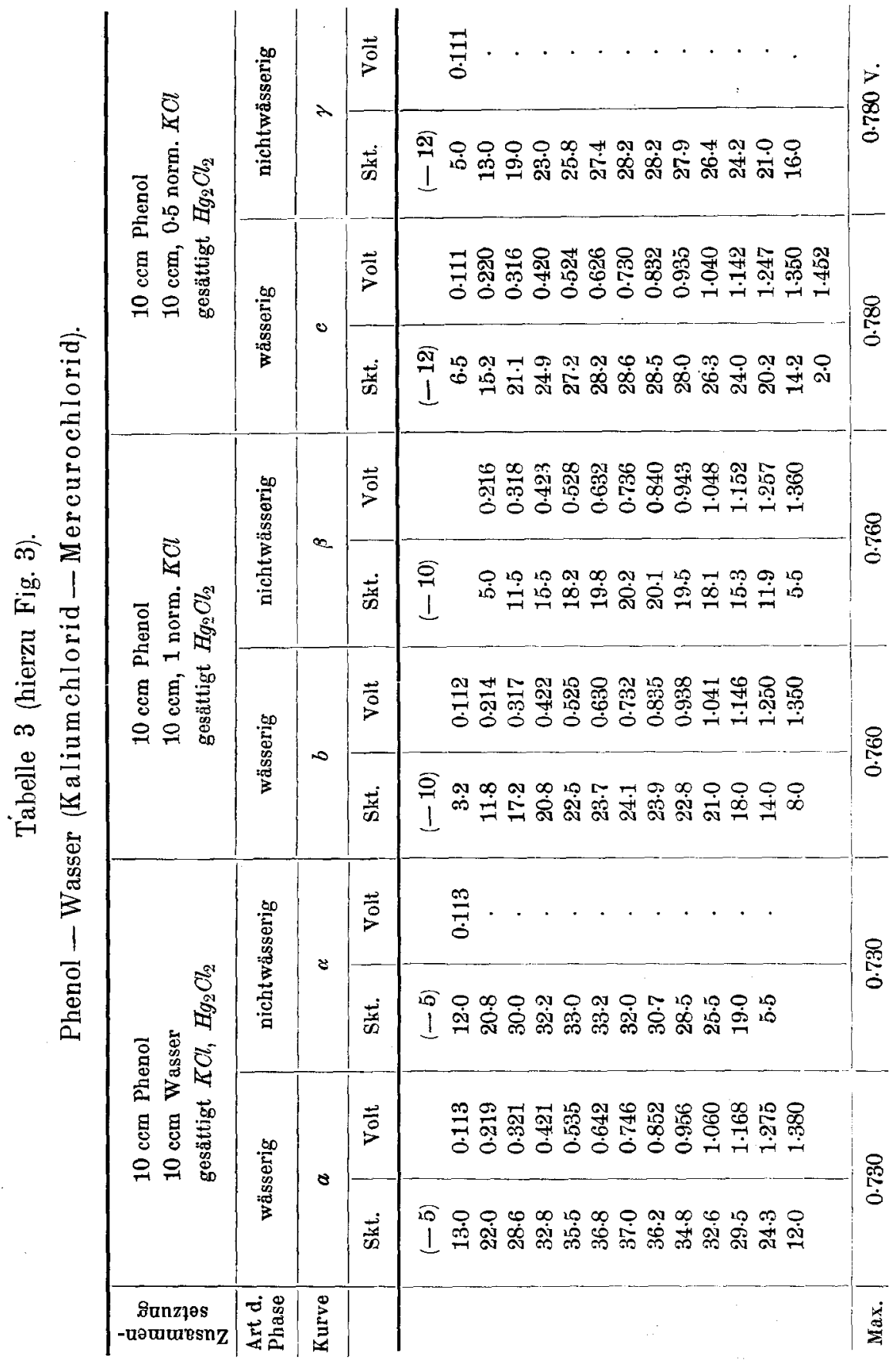


Elektrokapillarkurven in nichtwässerigen Lösungen.

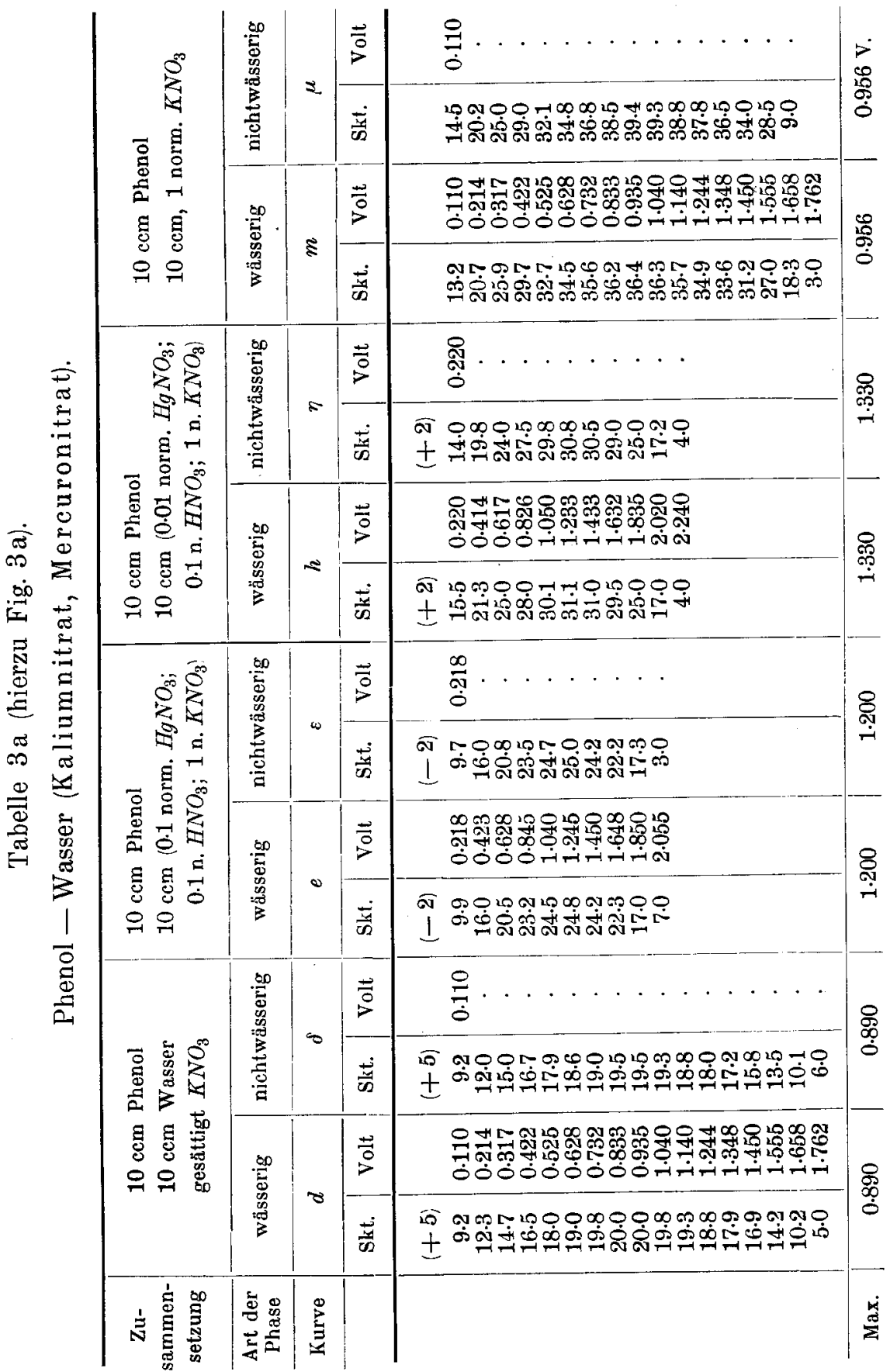




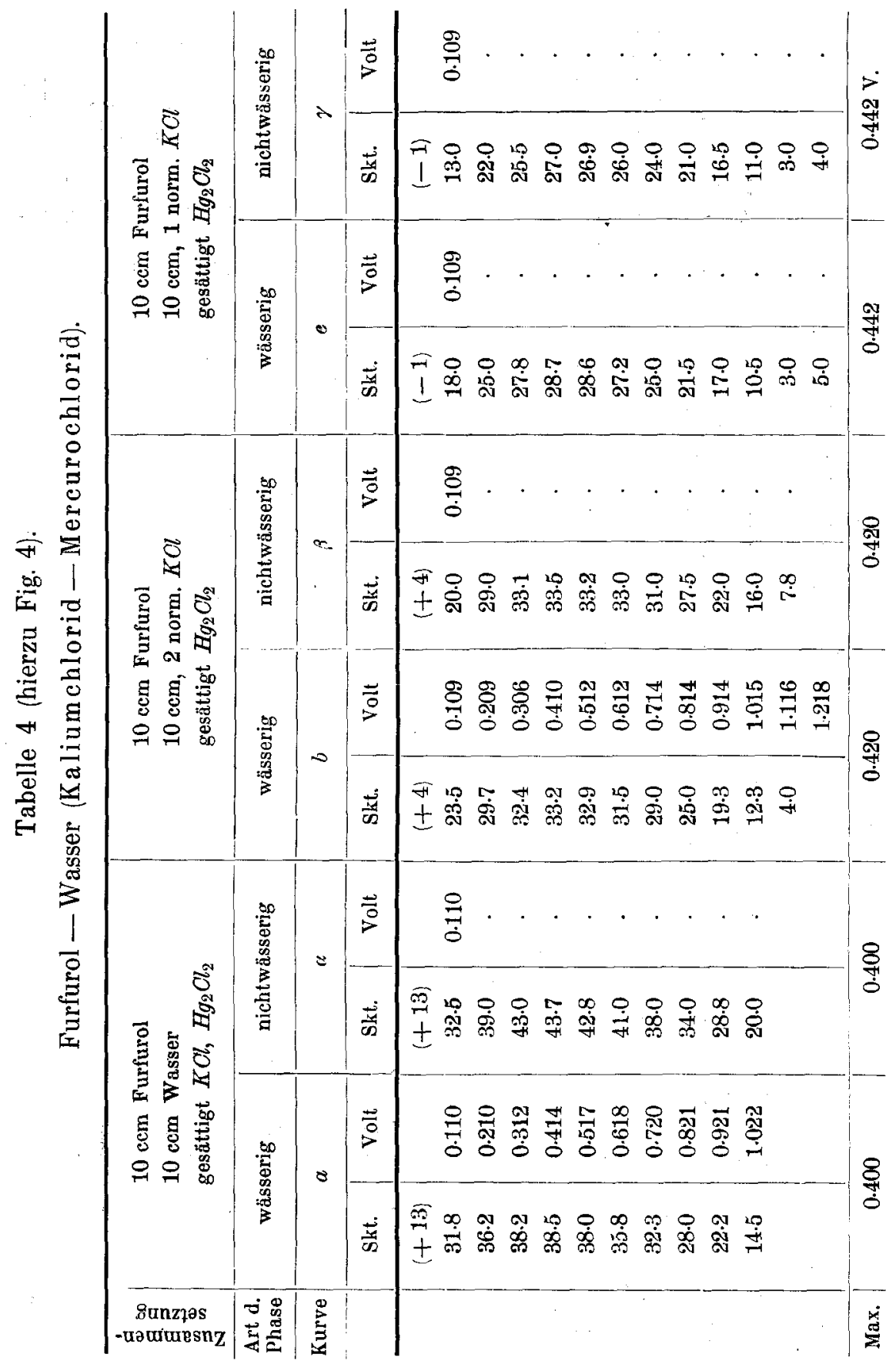




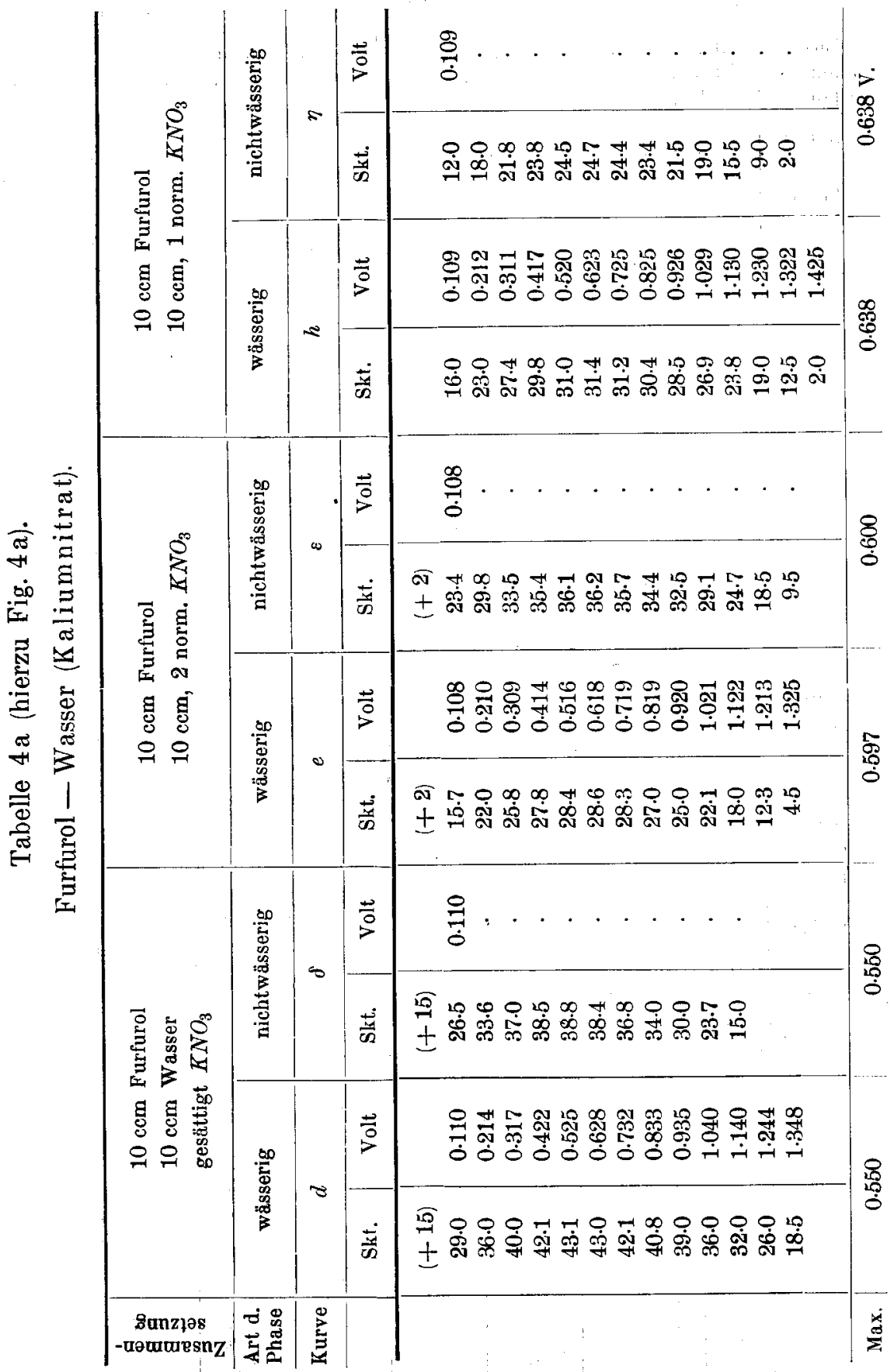


Tabelle 4b (hierzu Fig. 4b).

Furfurol - Wasser (Mercuronitrat, Kaliumnitrat).

\begin{tabular}{|c|c|c|c|c|c|c|c|c|}
\hline $\begin{array}{c}\text { Zu- } \\
\text { sammen- } \\
\text { setzung }\end{array}$ & \multicolumn{4}{|c|}{$\begin{array}{l}10 \mathrm{ccm} \text { Furfurol } \\
10 \mathrm{ccm}\left(0.1 \mathrm{n} . \mathrm{HgNO} \mathrm{O}_{3} ; 0.1 \text { n. } \mathrm{HNO}_{3} ;\right. \\
\left.\quad 1 \mathrm{n.} . \mathrm{KNO}_{3}\right)\end{array}$} & \multicolumn{4}{|c|}{$\begin{array}{l}10 \mathrm{ccm} \text { Furfurol } \\
10 \mathrm{ccm}\left(0.01 \mathrm{n} . \mathrm{HgNO} \mathrm{O}_{3} ; 0.1 \mathrm{n} . \mathrm{HNO}_{3} ;\right. \\
\left.\quad 1 \text { n. } \mathrm{KNO}_{3}\right)\end{array}$} \\
\hline $\begin{array}{c}\text { Art der } \\
\text { Phase }\end{array}$ & \multicolumn{2}{|c|}{ wässerig } & \multicolumn{2}{|c|}{ nichtwässerig } & \multicolumn{2}{|c|}{ wãsserig } & \multicolumn{2}{|c|}{ nichtwässerig } \\
\hline Kurve & \multicolumn{2}{|c|}{$m$} & \multicolumn{2}{|c|}{$\mu$} & \multicolumn{2}{|c|}{$t$} & \multicolumn{2}{|c|}{$\tau$} \\
\hline & Skt. & Volt & Skt. & Volt & Skt. & Volt & Skt. & Volt \\
\hline & $\begin{array}{l}: 2) \\
18.2 \\
24.0 \\
27.5 \\
29.7 \\
31.2 \\
32.0 \\
32.5 \\
32.6 \\
32.5 \\
32.1 \\
31.6 \\
30.9 \\
28.5 \\
27.0 \\
25.0 \\
22.9\end{array}$ & $\begin{array}{l}0.109 \\
0.212 \\
0.311 \\
0.417 \\
0.520 \\
0.623 \\
0.725 \\
0.825 \\
0.926 \\
1.029 \\
1.130 \\
1.230 \\
1.322 \\
1.425 \\
1.540 \\
1.641\end{array}$ & $\begin{array}{l}(: 2) \\
18.0 \\
23.8 \\
27.0 \\
29.0 \\
30.5 \\
31.3 \\
31.6 \\
31.8 \\
31.6 \\
31.2 \\
30.6 \\
29.8 \\
28.5 \\
26.8 \\
24.7 \\
22.2\end{array}$ & $\begin{array}{c}0.109 \\
: \\
: \\
: \\
: \\
\cdot \\
: \\
\cdot \\
\cdot \\
\cdot \\
\cdot \\
: \\
: \\
\cdot\end{array}$ & $\begin{array}{r}9.0 \\
14.5 \\
18.1 \\
20.3 \\
22.2 \\
23.4 \\
24.0 \\
24.2 \\
24.2 \\
23.8 \\
23.1 \\
22.1 \\
20.8 \\
19.2 \\
17.4 \\
15.0 \\
12.7\end{array}$ & $\begin{array}{l}0.110 \\
0.214 \\
0.317 \\
0.422 \\
0.520 \\
0.628 \\
0.732 \\
0.833 \\
0.934 \\
1.040 \\
1.140 \\
1.244 \\
1.348 \\
1.450 \\
1.555 \\
1.658 \\
1.762\end{array}$ & $\begin{array}{r}8.0 \\
12.5 \\
16.5 \\
18.9 \\
20.5 \\
21.5 \\
22.1 \\
22.4 \\
22.4 \\
22.0 \\
21.3 \\
20.2 \\
18.8 \\
16.8 \\
14.8 \\
12.3 \\
9.5\end{array}$ & $\begin{array}{c}0.110 \\
: \\
: \\
: \\
: \\
: \\
: \\
: \\
: \\
: \\
: \\
: \\
:\end{array}$ \\
\hline
\end{tabular}

Tabelle 5 (hierzu Fig. 5). Essigsäureäthylester - Alkohol - Wasser (Kaliumchlorid-Mercurochlorid).

\begin{tabular}{|c|c|c|c|c|c|c|c|c|}
\hline $\begin{array}{c}\text { Zu- } \\
\text { sammen- } \\
\text { setzung }\end{array}$ & \multicolumn{4}{|c|}{$\begin{array}{l}20 \mathrm{ccm} \text { Essigäther } \\
20 \mathrm{ccm} \text { Wasser } \\
20 \mathrm{ccm} \text { Äthylalkohol } \\
\text { gesättigt } \mathrm{KCl}, \mathrm{Hg}_{2} \mathrm{Cl}_{2}\end{array}$} & \multicolumn{4}{|c|}{$\begin{array}{l}20 \mathrm{ccm} \text { Essigäther } \\
20 \mathrm{ccm}, 2 \text { norm. } K \mathrm{Cl} \\
10 \mathrm{ccm} \text { Äthylalkohol } \\
\text { gesättigt } \mathrm{Hg}_{2} \mathrm{Cl}_{2}\end{array}$} \\
\hline $\begin{array}{l}\text { Art der } \\
\text { Phase }\end{array}$ & \multicolumn{2}{|c|}{ wässerig } & \multicolumn{2}{|c|}{ nichtwâsserig } & \multicolumn{2}{|c|}{ wässerig } & \multicolumn{2}{|c|}{ nichtwässerig } \\
\hline \multirow[t]{3}{*}{ Kurve } & \multicolumn{2}{|c|}{$a$} & \multicolumn{2}{|c|}{ a } & \multicolumn{2}{|c|}{$b$} & \multicolumn{2}{|c|}{$\beta$} \\
\hline & Skt. & Volt & Skt. & Volt & Skt. & Volt & Skt. & Volt \\
\hline & $\begin{array}{l}(-6) \\
15.0 \\
25.0 \\
32.8 \\
38.0 \\
43.0 \\
42.8 \\
41.9 \\
40.4 \\
38.3 \\
36.0 \\
33.0 \\
29.5 \\
25.2 \\
14.0\end{array}$ & $\begin{array}{l}0.068 \\
0.109 \\
0.157 \\
0.209 \\
0.306 \\
0.410 \\
0.512 \\
0.614 \\
0.714 \\
0.814 \\
0.914 \\
1.015 \\
1.116 \\
1.320\end{array}$ & $\begin{array}{r}(-6) \\
21.5 \\
28.5 \\
33.5 \\
36.6 \\
38.0 \\
37.5 \\
36.1 \\
34.3 \\
32.0 \\
29.0 \\
25.6 \\
21.3 \\
16.8 \\
2.2\end{array}$ & $\begin{array}{c}0.068 \\
: \\
: \\
: \\
: \\
: \\
: \\
: \\
: \\
: \\
:\end{array}$ & $\begin{array}{l}-4) \\
24.1 \\
30.7 \\
32.7 \\
32.0 \\
30.6 \\
28.4 \\
26.0 \\
23.0 \\
19.2 \\
15.2 \\
10.0 \\
3.3\end{array}$ & $\begin{array}{l}0.109 \\
0.209 \\
0.306 \\
0.410 \\
0.512 \\
0.614 \\
0.714 \\
0.814 \\
0.914 \\
1.015 \\
1.116 \\
1.218 \\
1.320\end{array}$ & $\begin{array}{r}(-4) \\
18.3 \\
28.4 \\
31.6 \\
31.5 \\
31.0 \\
30.0 \\
28.0 \\
25.2 \\
22.0 \\
18.7 \\
15.0 \\
10.8 \\
5.0\end{array}$ & $\begin{array}{c}0.109 \\
: \\
: \\
: \\
: \\
: \\
: \\
: \\
: \\
\cdot\end{array}$ \\
\hline Maximum & \multicolumn{2}{|c|}{0.328} & \multicolumn{2}{|c|}{0.328} & \multicolumn{2}{|c|}{0.316} & \multicolumn{2}{|c|}{$0.316 \mathrm{~V}$} \\
\hline
\end{tabular}




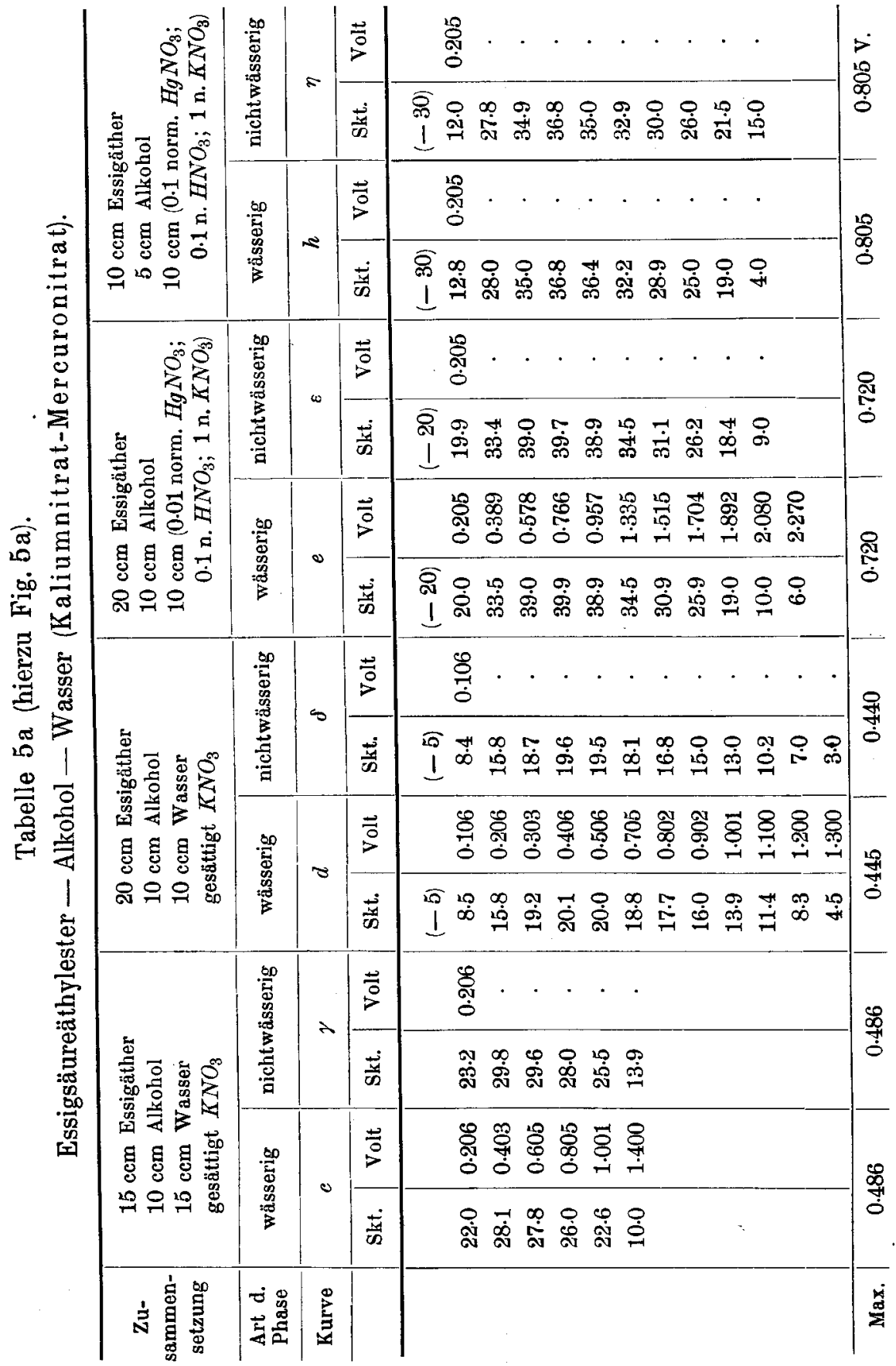




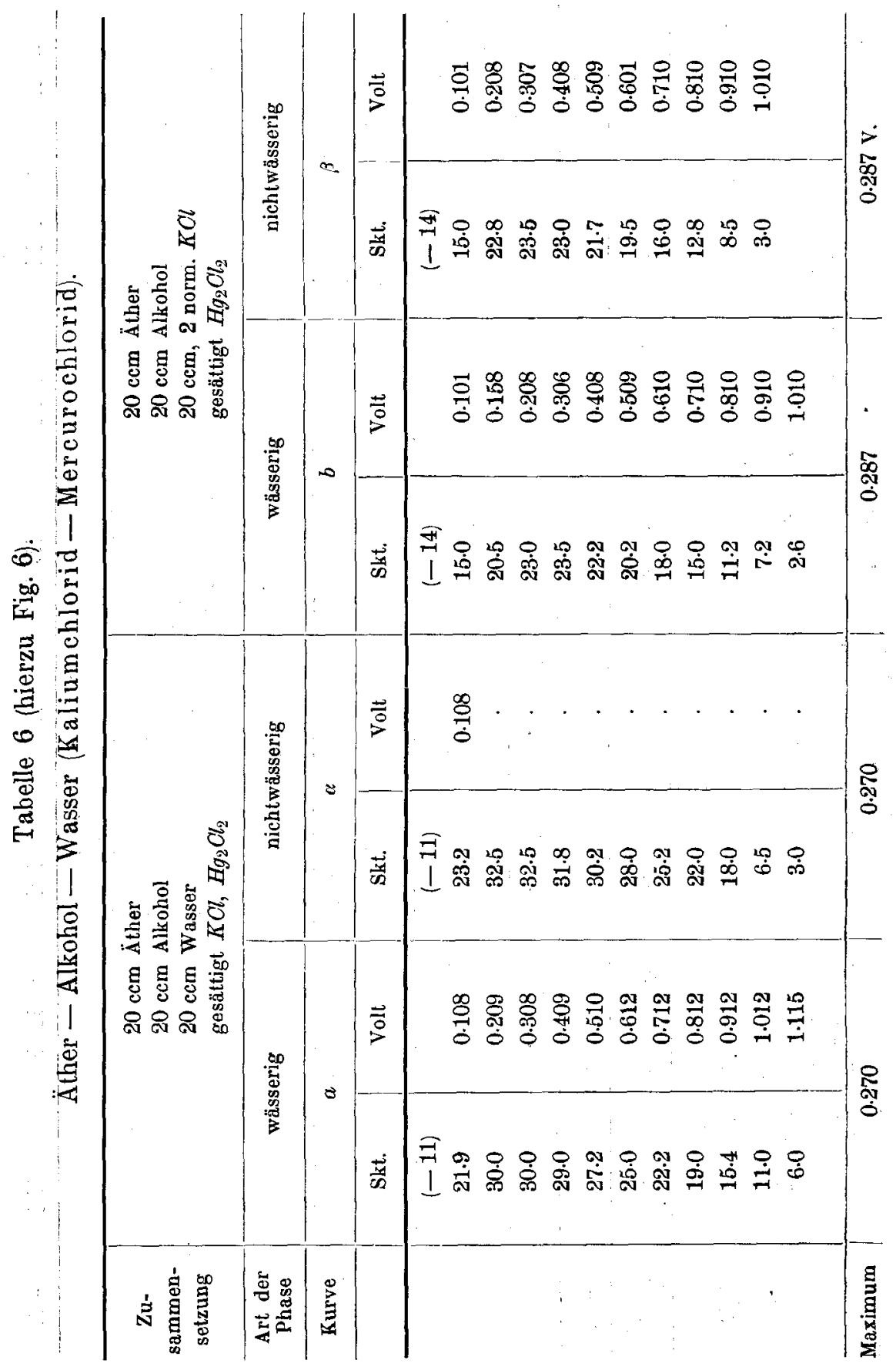


Elektrokapillarkurven in nichtwässerigen Lösungen.

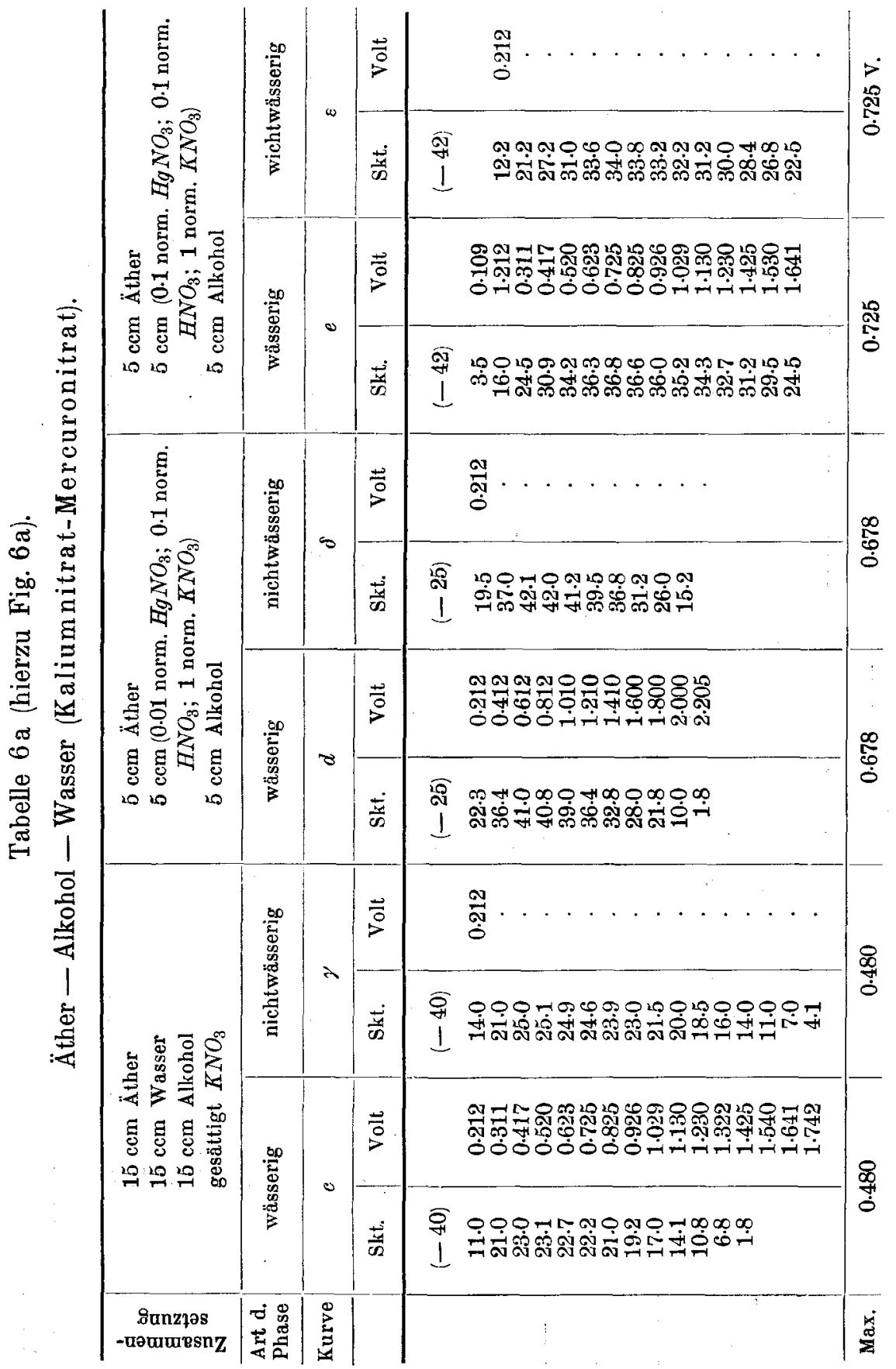




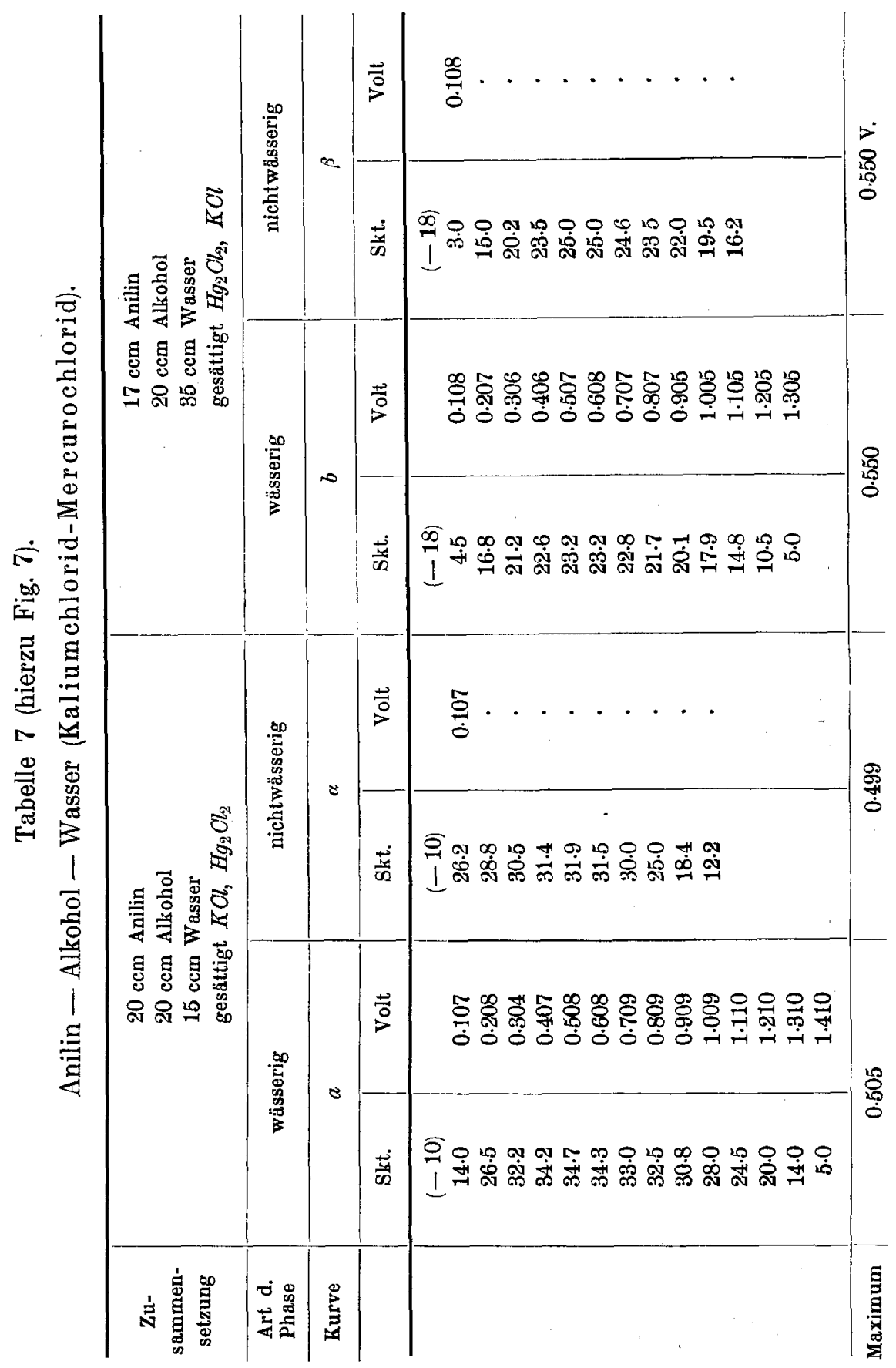




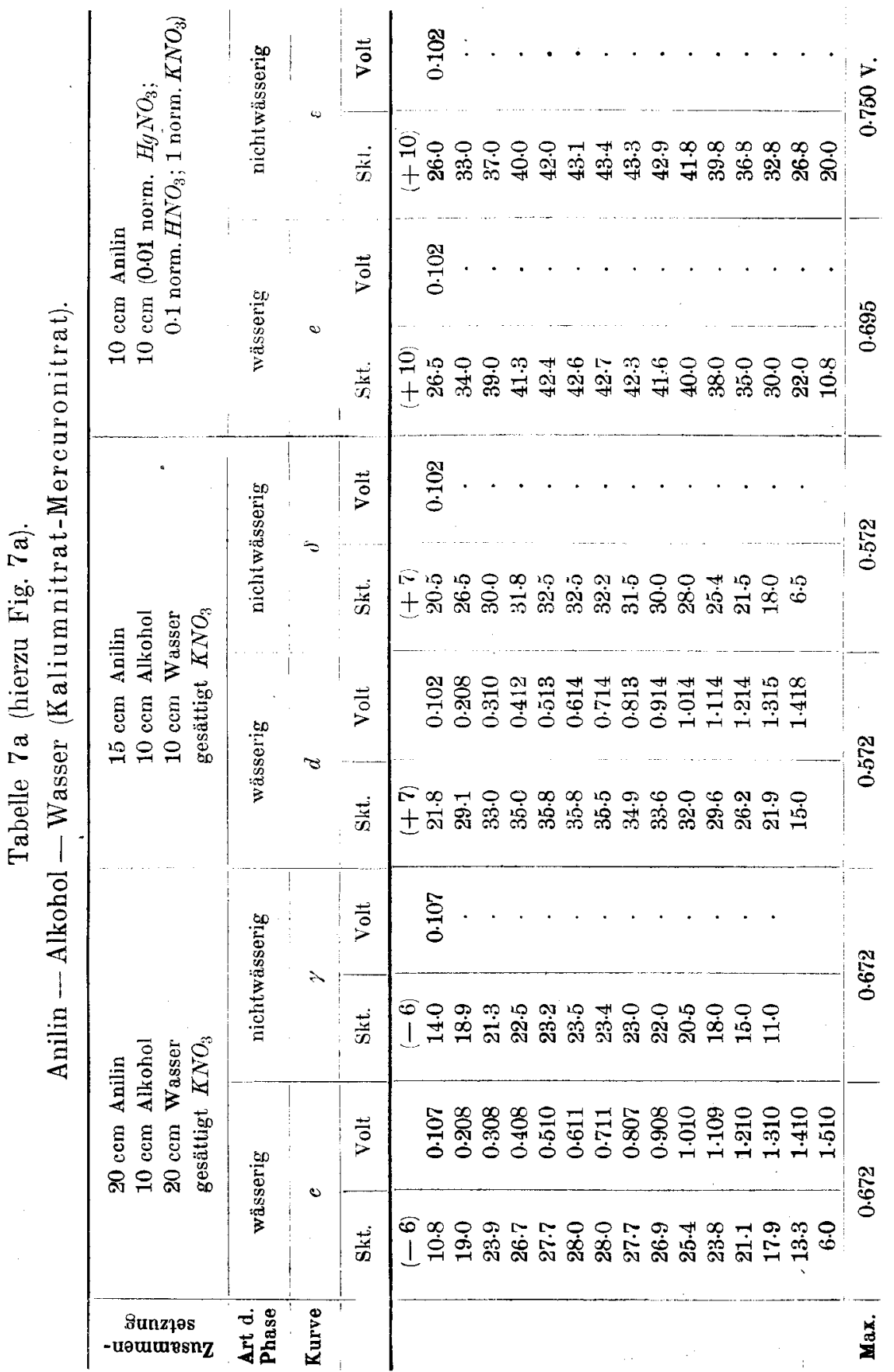

Keitschr. f. physik. Chemie. CIII. 


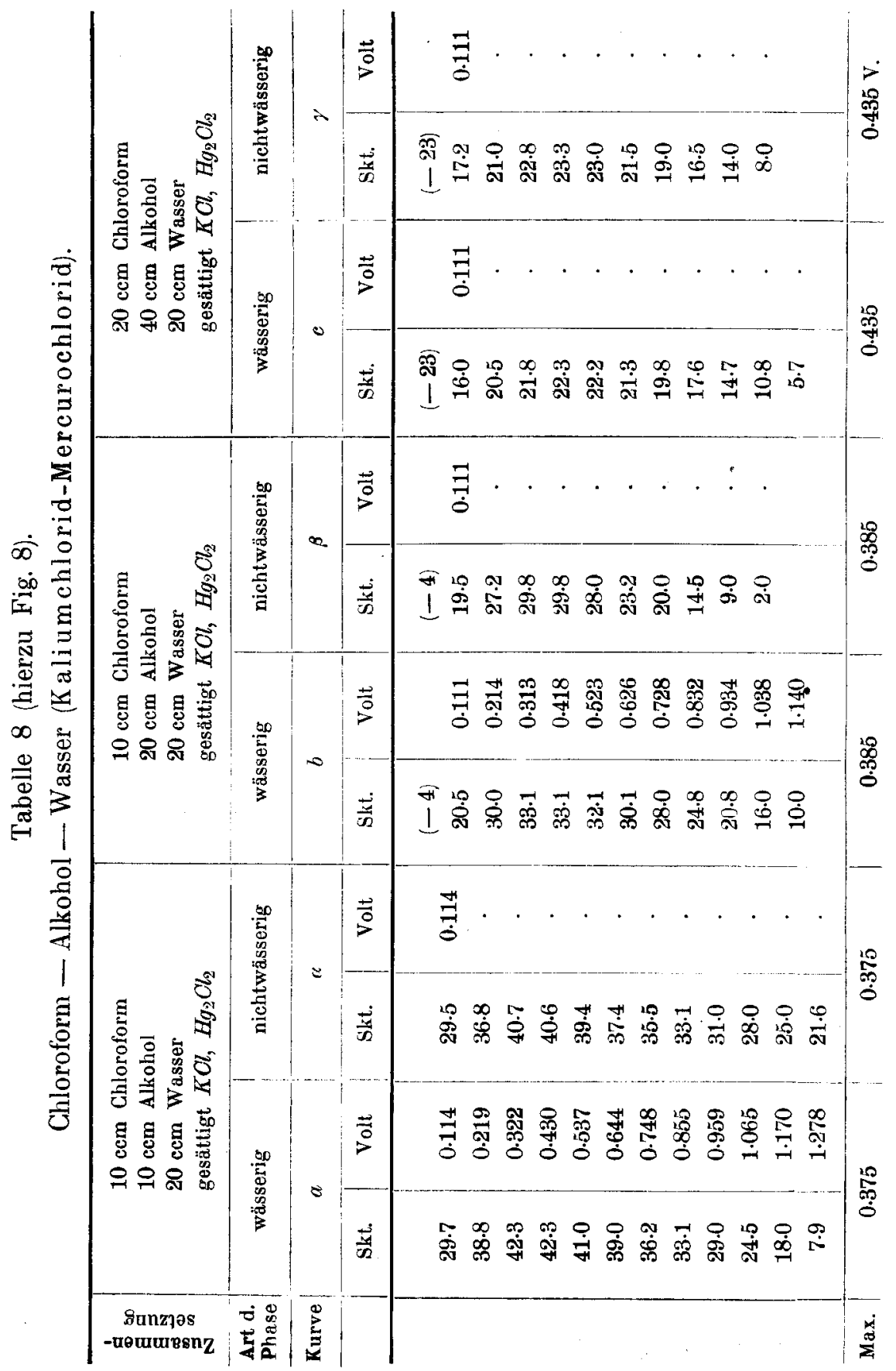




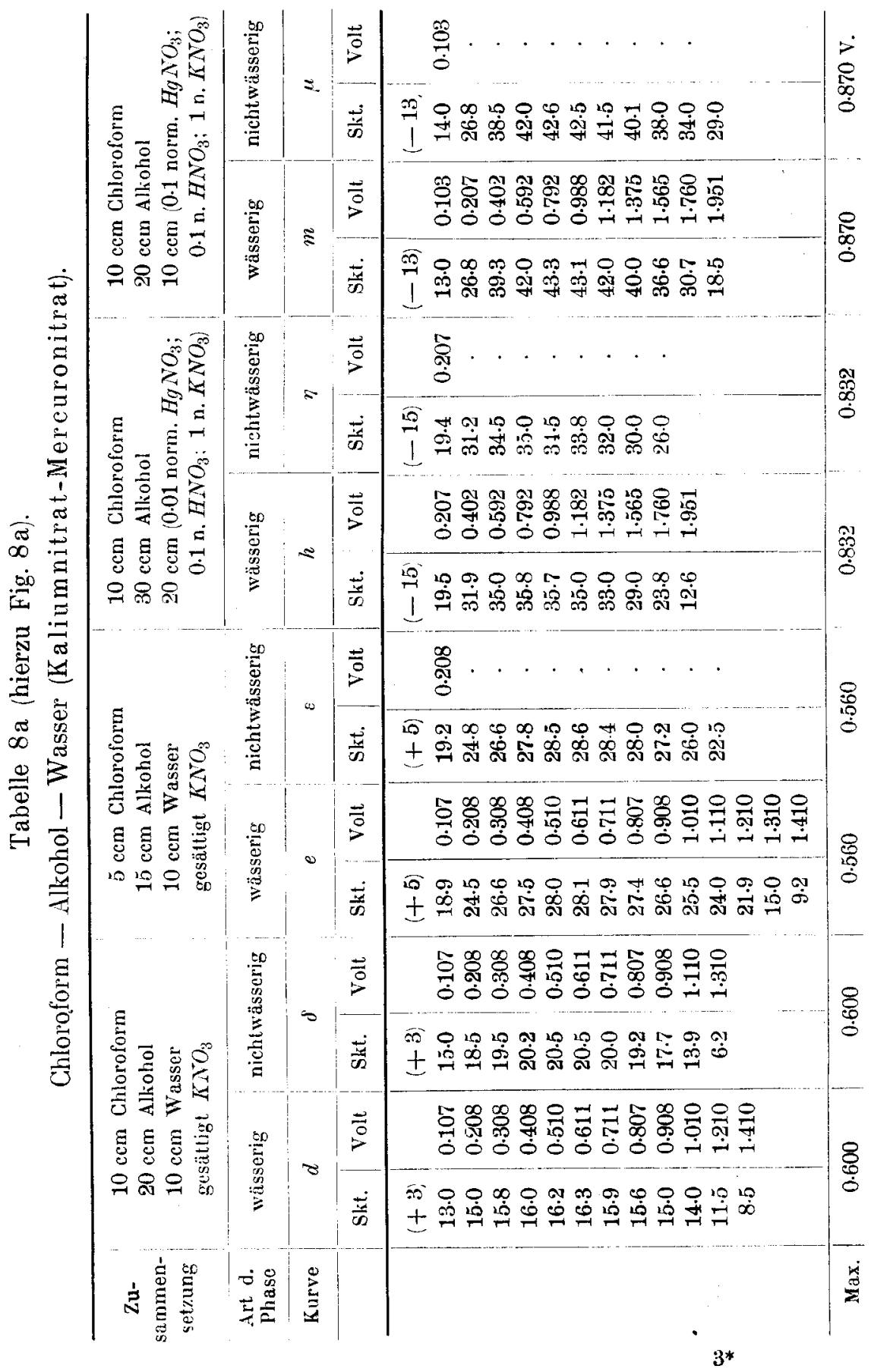


Tabelle 9 (hierzu Fig. 9).

Propylalkohol - Wasser (Kaliumchlorid-Mercurochlorid).

\begin{tabular}{|c|c|c|c|c|c|c|c|c|}
\hline $\begin{array}{l}\text { Zu- } \\
\text { sammen- } \\
\text { setzung }\end{array}$ & \multicolumn{4}{|c|}{$\begin{array}{l}20 \mathrm{ccm} \text { Propylalkohol } \\
30 \mathrm{ccm} \text { Wasser } \\
\text { gesättigt } K C l, H g_{2}\end{array}$} & \multicolumn{4}{|c|}{$\begin{array}{l}20 \mathrm{ccm} \text { Propylalkohol } \\
20 \mathrm{ccm} 2 \text { norm. } K \mathrm{KCl} \\
\text { gesättigt } \mathrm{Hg}_{2} \mathrm{Ol}_{2}\end{array}$} \\
\hline $\begin{array}{l}\text { Art d. } \\
\text { Phase }\end{array}$ & \multicolumn{2}{|c|}{ wässerig } & \multicolumn{2}{|c|}{ nichtwässerig } & \multicolumn{2}{|c|}{ wässerig } & \multicolumn{2}{|c|}{ nichtwässerig } \\
\hline \multirow{2}{*}{ Kurve } & \multicolumn{2}{|c|}{$a$} & \multicolumn{2}{|c|}{$\ll$} & \multicolumn{2}{|c|}{$b$} & \multicolumn{2}{|c|}{$\beta$} \\
\hline & Skt. & Volt & Skt. & Volt & Skt. & Volt & Skt. & Volt \\
\hline & $\begin{array}{c}(-25) \\
19.5 \\
29.9 \\
31.6 \\
31.3 \\
30.2 \\
28.9 \\
26.8 \\
24.0 \\
20.8 \\
17.0 \\
12.2 \\
6.0\end{array}$ & $\begin{array}{l}0.112 \\
0.216 \\
0.317 \\
0.424 \\
0.529 \\
0.634 \\
0.738 \\
0.841 \\
0.945 \\
1.049 \\
1.153 \\
1.258\end{array}$ & $\begin{array}{c}(-25) \\
19.3 \\
26.7 \\
28.0 \\
27.8 \\
26.5 \\
24.8 \\
22.3 \\
19.6 \\
16.1 \\
12.0 \\
6.7\end{array}$ & $\begin{array}{c}0.112 \\
\cdot \\
. \\
. \\
. \\
. \\
. \\
. \\
. \\
.\end{array}$ & $\begin{array}{c}(-15) \\
13.2 \\
19.1 \\
20.2 \\
20.0 \\
18.9 \\
17.0 \\
14.9 \\
12.9 \\
8.7 \\
4.5\end{array}$ & $\begin{array}{l}0.111 \\
0.217 \\
0.318 \\
0.425 \\
0.530 \\
0.635 \\
0.740 \\
0.844 \\
0.947 \\
1.052 \\
1.158\end{array}$ & $\begin{array}{c}(-15) \\
15.9 \\
22.0 \\
23.0 \\
22.9 \\
21.7 \\
20.0 \\
17.9 \\
14.9 \\
11.4 \\
7.0 \\
1.5\end{array}$ & $\begin{array}{c}0.111 \\
. \\
. \\
. \\
. \\
. \\
. \\
.\end{array}$ \\
\hline Maximum & \multicolumn{2}{|c|}{0.360} & \multicolumn{2}{|c|}{0.360} & \multicolumn{2}{|c|}{0.350} & \multicolumn{2}{|c|}{$0.350 \mathrm{~V}$} \\
\hline
\end{tabular}

Tabelle 10 (hierzu Fig. 10). Isobutylalkohol - Wasser - Alkohol (Kalium chlorid-Mercurochlorid).

\begin{tabular}{|c|c|c|c|c|c|c|c|c|}
\hline $\begin{array}{c}\mathrm{Zu}- \\
\text { sammen- } \\
\text { setzung }\end{array}$ & \multicolumn{4}{|c|}{$\begin{array}{l}10 \mathrm{ccm} \text { Isobutylalkohol } \\
10 \mathrm{ccm} \text { Alkohol } \\
20 \mathrm{ccm} \text { Wasser } \\
\text { gesättigt } \mathrm{KCl}, \mathrm{Hg}_{2} \mathrm{Cl}_{2}\end{array}$} & \multicolumn{4}{|c|}{$\begin{array}{l}20 \mathrm{ccm} \text { Isobutylalkohol } \\
10 \mathrm{ccm} \text { Alkohol } \\
20 \mathrm{ccm} \text { Wasser } \\
\text { gesättigt } \mathrm{KCl}, \mathrm{Hg}_{2} \mathrm{Cl}_{2}\end{array}$} \\
\hline $\begin{array}{l}\text { Art d. } \\
\text { Phase }\end{array}$ & \multicolumn{2}{|c|}{ wässerig } & \multicolumn{2}{|c|}{ niehtwässerig } & \multicolumn{2}{|c|}{ wässerig } & \multicolumn{2}{|c|}{ nichtwässerig } \\
\hline \multirow[t]{2}{*}{ Kurve } & \multicolumn{2}{|c|}{$a$} & \multicolumn{2}{|c|}{$r$} & \multicolumn{2}{|c|}{$b$} & \multicolumn{2}{|c|}{$\beta$} \\
\hline & Skt. & Volt & Skt. & Volt & Skt. & Volt & Skt. & Volt \\
\hline & $\begin{array}{c}(-15) \\
4.5 \\
19.0 \\
28.0 \\
30.1 \\
30.2 \\
29.5 \\
27.8 \\
25.8 \\
22.9 \\
19.2 \\
15.1 \\
10.0 \\
3.0\end{array}$ & $\begin{array}{l}0.112 \\
0.216 \\
0.317 \\
0.424 \\
0.529 \\
0.634 \\
0.738 \\
0.841 \\
0.945 \\
1.049 \\
1.153 \\
1.258 \\
1.363\end{array}$ & $\begin{array}{c}(-15) \\
6.6 \\
23.1 \\
30.7 \\
32.8 \\
33.2 \\
32.7 \\
31.6 \\
29.7 \\
27.5 \\
24.7 \\
21.1 \\
16.6 \\
10.6\end{array}$ & $\begin{array}{c}0.112 \\
. \\
. \\
. \\
. \\
. \\
. \\
. \\
. \\
. \\
. \\
. \\
.\end{array}$ & $\begin{array}{r}(-37) \\
7.0 \\
19.0 \\
22.0 \\
22.1 \\
21.4 \\
20.2 \\
18.6 \\
16.2 \\
13.5 \\
10.1 \\
6.0\end{array}$ & $\begin{array}{c}0.112 \\
. \\
. \\
. \\
. \\
. \\
. \\
\therefore \\
.\end{array}$ & $\begin{array}{c}(-37) \\
4.6 \\
16.6 \\
18.8 \\
19.1 \\
18.5 \\
17.4 \\
15.2 \\
12.8 \\
9.6 \\
5.6\end{array}$ & $\begin{array}{c}0.112 \\
\cdot \\
\cdot \\
\cdot\end{array}$ \\
\hline Maxinum & & & & & & & & $\mathrm{V}$ \\
\hline
\end{tabular}


Tabelle 11 (hierzu Fig. 11).

Wasser-Kaliumnitrat-Mercuronitrat.

\begin{tabular}{|c|c|c|c|c|c|c|c|c|}
\hline \multirow{3}{*}{$\begin{array}{l}\text { Zu- } \\
\text { sanimen- } \\
\text { setzung } \\
\text { Kurve }\end{array}$} & \multicolumn{2}{|c|}{$\begin{array}{l}0.01 \text { n. } \mathrm{Hg}_{2}\left(\mathrm{NO}_{3}\right)_{2} \\
0.1 \text { norm. } \mathrm{HNO}_{3} \\
1 \text { norm. } \mathrm{KNO} \mathrm{N}_{3}\end{array}$} & \multirow{2}{*}{\multicolumn{2}{|c|}{$\begin{array}{c}0.1 \text { n. } \mathrm{Hg}_{2}\left(\mathrm{NO}_{32}\right)_{2} \\
0.1 \text { norm. } \mathrm{HNO}_{3} \\
1 \text { norm. } K \mathrm{NO}_{3} \\
b\end{array}$}} & \multicolumn{2}{|c|}{1 norm. $K N O_{3}$} & \multirow{2}{*}{\multicolumn{2}{|c|}{$\begin{array}{c}0.1 \text { norm. } \mathrm{KNO}_{3} \\
d\end{array}$}} \\
\hline & \multicolumn{2}{|c|}{$a$} & & & \multicolumn{2}{|c|}{$c$} & & \\
\hline & Skt. & Volt & Skt. & Volt & Skt. & Volt & Skt. & Volt \\
\hline & $\begin{array}{r}(-2) \\
7.5 \\
14.0 \\
18 \cdot 0 \\
22 \cdot 6 \\
25 \cdot 8 \\
28.4 \\
30 \cdot 5 \\
32.0 \\
32.9 \\
32.9 \\
32 \cdot 1 \\
30.9 \\
29.0 \\
26 \cdot 3 \\
23 \cdot 5 \\
20 \cdot 0 \\
16 \cdot 3\end{array}$ & $\begin{array}{l}0.110 \\
0.215 \\
0.316 \\
0.424 \\
0.528 \\
0.623 \\
0.735 \\
0.837 \\
0.940 \\
1.042 \\
1.147 \\
1.250 \\
1.354 \\
1.460 \\
1.567 \\
1.668\end{array}$ & $\begin{array}{l}12.0 \\
18.9 \\
24.0 \\
28.5 \\
31.5 \\
34.8 \\
37.1 \\
39.0 \\
40.0 \\
40.5 \\
40.0 \\
39.0 \\
37.0 \\
34.6 \\
31.8 \\
28.5 \\
25.0 \\
20.5 \\
16.0\end{array}$ & $\begin{array}{l}0.110 \\
0.214 \\
0.317 \\
0.422 \\
0.525 \\
0.628 \\
0.732 \\
0.833 \\
0.935 \\
1.040 \\
1.140 \\
1.244 \\
1.348 \\
1.450 \\
1.555 \\
1.658 \\
1.762 \\
1.864 \\
1.968\end{array}$ & $\begin{array}{c}\langle+12\rangle \\
15.0 \\
19.8 \\
22.5 \\
24.5 \\
26.3 \\
27.2 \\
27.7 \\
27.7 \\
27.0 \\
25.8 \\
23.6 \\
21.1 \\
18.3 \\
15.0\end{array}$ & $\begin{array}{c}0.110 \\
: \\
: \\
: \\
: \\
: \\
: \\
: \\
: \\
: \\
:\end{array}$ & $\begin{array}{r}(+6) \\
9.0 \\
12.2 \\
15.8 \\
18.7 \\
21.0 \\
22.8 \\
23.5 \\
23.6 \\
23.1 \\
21.4 \\
19.8 \\
17.8 \\
15.0 \\
12.2 \\
9.0\end{array}$ & $\begin{array}{c}0.110 \\
: \\
: \\
: \\
: \\
: \\
: \\
\cdot \\
. \\
. \\
. \\
. \\
.\end{array}$ \\
\hline Maximum & & & & & & & & \\
\hline
\end{tabular}

\section{Das Ergebnis.}

Das Ergebnis der Untersuchung ist ein ganz eindeutiges: ohne Ausnahme wird gefunden, dass zwei im Verteilungsgleichgewicht stehende Lösungsphasen einen und densëlben Nernstschen Potentialsprung $\pi_{1}=\pi_{2}$ gegen ein gegebenes Metall besitzen. Dies gilt mit der Genauigkeit, mit der absolute Potentiale aus Elektrokapillarkurven abgeleitet werden können, also auf fünf bis zehn Millivolt. Mit demselben Grade der Genauigkeit lässt sich schliessen, dass die Phasengrenzkraft $\pi_{3}$ beim Verteilungsgleichgewicht null oder kleiner als fünf bis zehn Millivolt ist. Nachgewiesen ist dieses Verhalten für neun Lösungsmittelpaare und für vier Elektrolyte: $\mathrm{KCl}, \mathrm{KNO}_{3}, \mathrm{Hg}_{2}\left(\mathrm{NO}_{3}\right)_{2}$, $\mathrm{HNO}_{3}$, also für drei Kationen $\left(\mathrm{K}^{*}, \mathrm{Hg}, \mathrm{H}_{2}\right)$ und für zwei Anionen $\left(\mathrm{NO}_{3}^{\prime}, \mathrm{Cl}^{\prime}\right)$. Die Verallgemeinerung auf alle Lösungsmittel ist zweifellos gestattet; die Verallgemeinerung auf alle Ionen ist dagegen unsicher. 
Es könnte sein, dass die kapillaraktiven Ionen sich abweichend verhalten, dass also gerade dann, wenn ausgesprochene Ionenadsorptionspotentiale auftreten, zugleich noch ein Ionenverteilungspotentialsprung hinzukommt. Eine Entscheidung hierüber wird auf einem anderen, als dem in dieser Arbeit beschrittenen Wege gesucht werden müssen, da in diesem Fall die Ermittlung von $\pi_{1}$ und $\pi_{2}$ aus der Elektrokapillarkurve nicht angängig sein dürfte.

Vorliegende Arbeit wurde unter Leitung von Herrn Prof. Dr. E. Baur im Laboratorium für Elektro- und Physikalische Chemie der Eidgenössischen Technischen Hochschule in Zürich ausgeführt; und ich möchte nicht versäumen, an dieser Stelle meinem hochverehrten Lehrer für seine Anregungen und Ratschläge meinen herzlichsten Dank auszusprechen.

Zürich, den 18. Juli 1922. 


\section{Lebenslauf.}

Ich, J. Heinrich Wild, Sohn des Davids und der Babetta WildHiestand, von Mitlödi (Kanton Glarus), wurde am 3. Februar 1899 in Mitlödi geboren. Ich besuchte dort die Primarschule, nachher in Schwanden drei Jahre die Sekundarschule, worauf ich im Frühjahr 1914 in die „Evangelische Lehranstalt“ Schiers übertrat, wo ich mir im Herbst 1917 das Maturitätszeugnis erwarb.

Vom Wintersemester 1917 bis und mit Sommersemester 1921 studierte ich an der IX. Abteilung der Eidgenössischen Technischen Hochschule in Zürich, und erhielt im Sommer 1921 das Diplom als Fachlehrer der Naturwissenschaften in chemisch-physikalischer Richtung.

Vorliegende Arbeit wurde im Wintersemester 1921/22 und Sommersemester 1922 im Laboratorium für Elektro- und Physikalische Chemie der Eidgenössischen Technischen Hochschule ausgeführt. 